\title{
Meeting abstracts of Colossal Facet Conference - 2nd World Congress on Cancer 2018 "Oncology and Cancer therapeutics in the 21st century"
}

\author{
Bologna, Italy; 23-25 Jul 2018; Published: 18 Apr 2019 \\ Correspondence to: Dr. Ciro Isidoro, Department of Health Sciences, Università del Piemonte Orientale "A. Avogadro", Novara \\ 28100, Italy. E-mail: ciro.isidoro@med.uniupo.it
}

\section{Editorial note}

This Special Issue of Journal of Cancer Metastasis and Treatment is dedicated to the proceedings of the 2nd World Congress on Cancer 2018 held in Bologna (Italy) in July 23-25. The theme of the Conference Cancer-2018 was "Oncology and Cancer therapeutics in the 21st century".

Goal of the meeting was to share the foremost updated knowledge on the biology and the novel therapeutic options in Cancer. The conference focused on the mechanisms of cancer development, on the relationship between tumor microenvironment, metabolism and Cancer progression, novel technologies for early detection, and on novel pharmacological approaches to treat cancer patients. Major topics included epigenetics, inflammation, drug resistance, cancer stem cells, autophagy, cell metabolism, experimental models, biobanking, immune response, cachexia, molecular diagnosis, biomarkers, patient clinical care and complementary phytotherapy.

This Special Issue will cover the researches at the cutting edge in the study of the biology and clinical care of Cancer presented at the Conference.

Invited Speakers, Poster presenters and participants can contribute to this special issue with either an extended abstract (no page limit), Commentary, Original articles and (mini) review articles. Inclusion of schemes, Figures and Cartoon summarizing the results is strongly encouraged. An "ad hoc" editorial team will oversee the peer review of research and review articles.

Ciro Isidoro, Guest Editor

Keywords: Autophagy, biobanking, biomarkers, cachexia, cancer metabolism, cancer therapy, cell death, drug resistance, epigenetics, metastasis

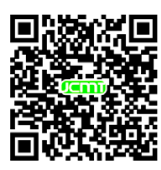




\title{
1. Autophagy in cancer: epigenetic regulation and therapeutic opportunities
}

\author{
Ciro Isidoro, Chiara Vidoni, Alessandra Ferraresi, Eleonora Secomandi, Letizia Vallino
}

Department of Health Sciences, Università del Piemonte Orientale, Italy, Novara 28100, Italy.

Autophagy, lysosome-effected degradation pathway that eliminates damaged and redundant cellular selfconstituents, plays a pivotal role in maintaining cell homeostasis. Autophagy is a stress-response triggered under nutrient- and energy-restricted situations, as well as in response to damaging and harmful injuries. Autophagy play an important role in preventing tumorigenic transformation by cooperating with the systems for the quality control of the proteome and of the genome. Defective autophagy may facilitate carcinogenesis, and in cancer cells autophagy may serve a survival function allowing to cope with damages induced by anti-cancer treatments or with the lack of oxygen and nutrients. The composition of the tumour environment impacts on autophagy in cancer cells. In fact, the metabolic cross-talk with stromal cells and their soluble factors, including inflammatory cytokines, can modulate the availability of nutrients, energy and growth factors that eventually modulate autophagy. Autophagy in cancer cells is also modulated epigenetically by changes of the chromatin structures and the presence of microRNA. The fact that autophagy constitutes the integrated response to all metabolic stresses and that can be epigenetically modulated offers the possibility to treat cancer through the use of autophagy-targeted epigenetic modifiers.

\section{Biography}

Ciro Isidoro is Professor of Pathology at the School of Medicine of Università del Piemonte Orientale (Novara, Italy). He received his doctoral degree in Biological Sciences from the University of Torino (Italy) and his doctoral degree in Medicine and Surgery from the University of Piemonte Orientale (Novara, Italy). He is Visiting Professor at the Faculty of Medicine, Siriraj Hospital, of Mahidol University (Bangkok, Thailand), Visiting Professor at the Department of Cell Biology of the Oklahoma City University Health Sciences Center (US), and Professeur Honoraire at the Faculté de Medecine et de Pharmacie de l'Université de Franche-Comté, Besancon (France). He is member of the Scientific board of the «Integrative Cancer Research Center of the Georgia Institute of Technology» (Atlanta, US). Ciro Isidoro has co-authored > 120 peer-reviewed original articles published in international journals. He serves as Co-Editor in Chief of the Journal of Traditional and Complementary Medicine and Associate Editor of Autophagy, Molecular Carcinogensis, BMC Cancer, and other journals. His fields of expertise include the subjects "autophagy regulation in cancer" and "mechanisms of anticancer activity of dietary products".

\section{Understanding parasites in the United States}

\section{Omar M. Amin}

Parasitology Center Inc, Scottsdale, AZ 85259, USA.

This Power Point presentation is based on our work at Parasitology Center, Inc. (PCI), in Scottsdale, Arizona, USA and covers the diagnosis, pathology, and treatment of human parasitic infections in the United States based on our own patient history and cases diagnosed. A brief introduction to laboratory procedures, misdiagnoses/mistreatment, and impact on public health is made. The presentation begins with a quiz which is answered at the end. A systematic treatment of protozoan, helminth (worm), and arthropod parasites follows, emphasizing epidemiology and exposure, symptoms, gross pathology, and herbal and allopathic remedies including our own anti-parasitic herbal product Freedom/Cleanse/Restore. 
All topics are presented with illustrated and labeled pictures of the various kinds of parasites and their gross pathology in human tissues, when applicable. The presentation is followed by a brief discussion of case histories as tested by us at PCI as well as by a treatment of intestinal pathogenic bacteria, also tested by us, that usually cause GI symptoms similar to those caused by intestinal parasites.

\section{The new oncological theory and its importance in the war with cancer}

\section{Andrei P. Kozlov}

Biomedical Center, Peter the Great St.Petersburg Polytechnic University and Research Institute of Pure Biopreparation, St. Petersburg 195251, Russia.

Earlier I formulated the concept of the possible evolutionary role of tumors (A.P. Kozlov, "Evolution by Tumor Neo functionalization", Elsevier/Academic Press, 2014). This concept suggests that heritable tumors at earlier stages of progression supply evolving multicellular organisms with extra cell masses for the expression of newly evolving genes. After expression of novel genes in tumor cells, tumors differentiate in new directions and give rise to new cell types, tissues and organs.

In the presentation, the bulk of data supporting the positive evolutionary role of tumors will be reviewed, obtained both in the lab of the author and from the literature sources. The new concept supports the possibility of sustaining a stable tumor mass as a strategy in cancer therapy.

As a result of experimental confirmation of nontrivial predictions of the new concept a new class of genes - Tumor Specifically Expressed, Evolutionarily New (TSEEN) genes - was discovered. Examples of TSEEN genes will be presented. TSEEN genes may represent the new targets in the war with cancer.

\section{Discovery of a novel PRMT5 inhibitor to treat pancreatic and colorectal cancers}

\section{Tao Lu}

Department of Pharmacology and Toxicology, Indiana University School of Medicine, 635 Barnhill Drive, Indianapolis, IN 46202, USA.

Recently, we disocvered that protein arginine methyltransferase 5 (PRMT5) functions as a novel activator of the nuclear factor $\kappa \mathrm{B}(\mathrm{NF}-\mathrm{\kappa B})$, a culprit in pancreatic ductal adenocarcinoma (PDAC) and colorectal cancer (CRC). In this study, we adapted the AlphaLISA technique into a PRMT5-specific high throughput screen, and discovered PR5-LL-CM01 as a novel small molecule inhibitor of NF- $\kappa$ B. Treatment of PDAC and CRC cells with PR5-LL-CM01 inhibited tumor cell proliferation, anchorage-independent growth, as well as cell migration. Furthermore, PR5-LL-CM01 greatly impeded tumor growth in PDAC and CRC in vivo xenograft models. Importantly, PR5-LL-CM01 exhibited more potent anti-tumor effect than the commercial PRMT5 inhibitor, EPZ015666, in both PDAC and CRC. Our study has established PR5-LL$\mathrm{CM} 01$ as a potential basis for novel drug development to treat PDAC and CRC in the future.

\section{Biography}

Dr. Tao Lu is a tenure-track Assistant Professor and principle investigator at Department of Pharmacology and Toxicology, and a member of Simon Cancer Center at Indiana University School of Medicine. She 
obtained her Ph.D. degree from University of Toledo, School of Medicine, and finished her postdoctoral training with the world renowned scientist Dr. George Stark at Cleveland Clinic, Ohio. Her research focuses on the discovery of novel regulators of NF- $\mathrm{B}$, particularly, on the epigenetic regulation of NF- $\mathrm{B}$ and its role in cancer therapeutics. She won multiple awards at international scientific meetings. Dr. Lu has published near 50 papers with 2 were highlighted by F1000 Prime. She currently holds 2 provisional patents regarding NF- $\mathrm{\kappa B}$ regulation and serves as the editorial board member of 8 scientific journals.

\title{
5. Using a multiplexed immunofluorescence assay to uncover an immunosuppressive mechanism of tumor-associated macrophages in the pancreatic tumor microenvironment
}

\author{
Anna Juncker-Jensen, Jun Fang, Judy Kuo, Mate Nagy, Qingyan Au, Eric Leones, Flora Sahafi, Raghav \\ Padmanabhan, Nicholas Hoe, Josette William
}

NeoGenomics, Aliso Viejo, CA 92656, USA.

Background: Pancreatic ductal adenocarcinoma (PDAC) is characterized by an excessive amount of desmoplastic stroma seeded with inflammatory cells and it is one of the most aggressive forms of cancer with no current specific therapies. Tumor-associated macrophages (TAMs) are a major component of the tumor microenvironment (TME), and in most solid cancers increased TAM infiltration is associated with a poor prognosis. TAMs can be described as classically activated M1 types with pro-inflammatory antitumor functions, vs. alternatively activated M2 types with immunosuppressive pro-tumor functions. The immunosuppressive functions of M2 TAMs can be exerted through release of cytokines and growth factors as well as via direct recruitment of T regulatory cells (Tregs), a subset of lymphocytes responsible for immune tolerance of the system to the tumor. While the differentiation from M1 to M2 in PDAC has been shown to be associated with a worse prognosis ${ }^{[1]}$, not much is known about PDAC TAM polarization and its potential correlation to Treg recruitment.

Methods: For this study we have used MultiOmyx, a proprietary, multiplexing assay with similar staining characteristics as standard IHC stains but with the significant advantage that up to 60 protein biomarkers can be interrogated from a single FFPE section ${ }^{[2]}$. MultiOmyx protein immunofluorescence assays utilize a pair of directly conjugated Cyanine dye-labeled (Сy3, Cy5) antibodies per round of staining. Each round of staining is imaged and followed by novel dye inactivation chemistry, enabling repeated rounds of staining and deactivation.

Results: Using the pan macrophage marker CD68 in combination with either M1 marker HLA-DR or M2 marker CD163 we confirmed the presence of M1 (CD68+HLA-DR+) and M2 (CD68+CD163+) populations in 9 stage IIB non-metastatic PDAC FFPE samples, the vast majority being of the M2 subtype. Moreover, we found a positive significant correlation (Pearson's correlation $P<0.05$ ) between the presence of M2 TAMs and Tregs (CD3+CD4+FoxP3+), but not between M1 TAMs and Tregs. Moreover, in a spatial nearest neighbor analysis we found M2 type TAMS to be in closer proximity to Tregs compared to M1 type TAMs.

Conclusion: We demonstrate a positive significant correlation between the presence of M2 TAMs and Tregs in the TME of PDAC, suggesting a possible pathway in which TAM polarization plays an immunosuppressive function by recruiting Tregs. PDAC is one of the most aggressive forms of cancer with a 5-year survival rate below 5\% and no current specific therapies. An increasing number of studies show accumulation of immune suppressor cells such as MDSCs and TAMs in PDAC patients. Hopefully, 
a greater understanding of the phenotypes and functions of subsets of these cell types, will result in new cancer immunotherapy strategies for PDAC.

\section{REFERENCES}

1. Kurahara H, Shinchi H, Mataki Y, Maemura K, Noma H, et al. Significance of M2-polarized tumor-associated macrophages in pancreatic cancer. J Surg Res 2011;167:e211-9.

2. Gerdes MJ, Sevinsky CJ, Sood A, Adak S, Bello MO, et al. Highly multiplexed single-cell analysis of formalin-fixed, paraffin-embedded cancer tissue. Proc Natl Acad Sci U S A 2013;110:11982-7.

\section{Stereotactic radiosurgery for brain metastasis: the end of whole brain radiotherapy?}

\section{Leonardo Frighetto ${ }^{1,2}$}

${ }^{1}$ Department of Neurosurgery, Radiotherapy and Radiosurgery Unit, Hospital Moinhos de Vento, Porto Alegre, RS 90035-004, Brazil.

${ }^{2}$ Coordinator, Neurosurgery Residency Program, Federal University of South Frontier, Zona Rural, Realeza 85770-000, Brazil.

The objective of this presentation is to evaluate the applications of stereotactic radiosurgery (SRS) alone or in association with whole brain radiotherapy (WBRT) in the management of metastatic brain disease. Special attention was given to the influence of these treatment modalities on intracranial progression, local control, functional preservation, quality of life and survival. WBRT alone or following microsurgery has been the gold standard treatment of brain metastasis. The limited disease concept of oligometastatic brain disease, opened space to the use of focal therapies, such as SRS. Furthermore, delaying neurocognitive and cerebelar deterioration related to WBRT, is a worthwile goal in brain metastasis patients. Moreover, SRS is more effective in the treatment of tumors considered resistant to WBRT such as melanoma and renal cell carcinoma. Analysis of the available data, including prospective randomized control trials in which SRSalone was compared with WBRT+SRS, for patients with 1-4 brain metastasis, have proved that there was no significant difference in survival, neurologic death, functional independence and quality of life between the two treatment modalities. The omission of WBRT significantly increased the intracranial progression in new sites, requiring frequent monitoring in order to detect new lesions before they became symptomatic. Level 1 data has also demonstrated better neurocognitive function in patients not submitted to WBRT. The favorable outcomes demonstrated with SRS, have made possible its application in the focal treatment of surgical cavities. Therefore, the use of the SRS alone approach for oligometastatic brain disease may allow the majority of patients to avoid WBRT.

\section{Biography}

Leonardo Frighetto is the scientific coordinator of the Stereotactic Radiosurgery Unit at Moinhos de Vento Hospital and coordinator of the Neurosurgery Residency at the Federal University of the South Frontier, in Brazil. After finishing a fellowship at the Department of Stereotactic and Functional Neurosurgery at the University of California Los Angeles, he participated in the development of one of the first Stereotactic Radiosurgery Units in Brazil. The hospital is accredited by the Joint Commission International and affiliated to Johns Hopkins Medicine International. He also served as the President of the Brazilian Society of Radiosurgery from 2012 to 2014. 


\title{
7. Role of ERCC5 polymorphism and risk of breast cancer in Thailand
}

\author{
Malinee Pongsavee ${ }^{1}, K^{K a m o l ~ W i s u w a n ~}{ }^{2}$
}

\author{
${ }^{1}$ Department of Medical Technology, Faculty of Allied Health Sciences, Thammasat University, Patumthani \\ 12121, Thailand. \\ ${ }^{2}$ Department of Pathology, Ubon Ratchathani Cancer Hospital, Ubon Ratchathani 34000, Thailand.
}

Breast cancer is a major public health problem around the world, including Thailand and it has the highest ranking among female cancer. Polymorphism of ERCC5 gene (excision repair cross-complementary group 5 gene or ERCC5) was reported to associate with an increased risk of breast cancer. This study aims to investigate the relationship between ERCC5 polymorphism and the breast cancer risk in the lower northeastern region women of Thailand. One hundred fifty samples from breast cancer patients and 122 samples from healthy control group were analysed. Genomic DNA was extracted from white blood cell of all samples. The real-time polymerase chain reaction was used to demonstrate genetic polymorphism of ERCC5. The results showed that the ERCC5 rs751402 polymorphism variant AG was associated with an increased risk of breast cancer $(P<0.05)$. This study demonstrated that ERCC 5 rs751402 genotype AG was associated with breast cancer risk in the lower northeastern region women of Thailand.

\section{The RNA Disruption Assay as a tool to predict pathologic complete response and improved disease-free survival in breast cancer patients after neoadjuvant chemotherapy}

\author{
Amadeo Mark Parissenti ${ }^{1,2,3,4,5}$ \\ ${ }^{1}$ Health Sciences North Research Institute, Sudbury, ON P3E 5J1, Canada. \\ ${ }^{2}$ Laurentian University, Sudbury, ON P3E 2C6, Canada. \\ ${ }^{3}$ The Northern Ontario School of Medicine, Sudbury, ON P3E 2C6, Canada. \\ ${ }^{4}$ Rna Diagnostics, Inc., Sudbury, ON P3E 2H3, Canada. \\ ${ }^{5}$ Rna Diagnostics, Inc., Toronto, ON M4T 1L9, Canada.
}

We have observed that a variety of mechanistically distinct chemotherapy drugs can induce the degradation of ribosomal RNA into high molecular weight fragments in multiple tumour cell lines in a dose- and time-dependent manner - a phenomenon we term "RNA disruption". Moreover, we have developed the RNA disruption assay (RDA) to quantify ribosomal RNA degradation in tumour cell lines and tissues. Interestingly, chemotherapy agents cannot induce RNA disruption in chemo-resistant cell lines, suggesting RDA may be useful clinically in monitoring tumour response to chemotherapy in real time. Consistent with this view, we observed that high mid-treatment tumour RNA disruption in patients with locally advanced breast cancer is associated with a pathologic complete response (pCR) and improved disease-free survival after epirubicin/docetaxel neoadjuvant chemotherapy. Moreover, we and our collaborators have recently shown that high tumour RNA disruption after only one cycle of neoadjuvant trastuzumab-based chemotherapy predicted for a post-treatment pCR in patients with Her2+ breast cancer. The BREVITY clinical trial (Breast Tumour Response Evaluation for Individualized Therapy) will soon be accruing patients in Germany, Italy, Ireland, the United States, and Canada to further assess the utility of RDA to predict response and survival after neoadjuvant chemotherapy. The patient eligibility criteria, trial design, timing of tumour biopsies, and both primary and secondary study endpoints will be discussed. RDA may enable oncologists to identify patients with chemo-resistant tumours early in neoadjuvant chemotherapy, 
reducing the toxic side-effects of the ineffective regimen, and enabling patients to move to potentially more beneficial downstream or alternative treatments.

\section{Biography}

Dr. Parissenti is a professor of biochemistry at Laurentian University and the Northern Ontario School of Medicine in Sudbury, ON Canada. The RNA Disruption Assay was developed through Dr. Parissentis collaborations with clinical researchers from the Canadian Cancer Trials Group and Rna Diagnostics, Inc. More information on RNA disruption and RDA can be obtained at http://rnadiagnostics.com.

\section{ULLB-0005, a novel protein for cancer treatment}

\section{Sudeep Kumar}

Unichem Laboratories Ltd, Pilerne Industrial Estate, Goa 403511, India.

Recombinant protein derived from a natural fungal protein, which has high binding specificity toward the carbohydrate antigen. The natural Amino acid sequence has been modified to make more stable and soluble protein. Modified sequence has been cloned and express in E. coli. The protein was purified through different column chromatography and was characterized as a single protein. Promising cytotoxicity was observed in different cancer cell line, with a good safety profile in human PBMCs with ULLB-0005. The efficacy of the Molecule as antitumor agent was assessed in respective xenograft immuno-compromised mice models in vivo. As expected the molecule showed strong anti-cancer activity in immune-compromised mice model in various cancers which was observed in the reduction of tumor volume. Mechanistic study showed strong apoptotic signal by modulating phosphatidyl serine externalization, mitochondrial membrane depolarization, cell cycle arrest, ultimately leading to death in cancer cells. Inhibition of proliferation and migration was observed in human endothelial cells, suggesting potential antiangiogenic effect. Single dose Pharmacokinetic study was performed for the molecule in BALB/C MICE with i.v., i.p. and i.m. route.

To elaluate this novel protein as a combination with chemotherapy further studies was conducted and found that the molecule showed good synergy in in vitro with approved chemotherapeutic agents for Breast and Pancreatic cancer. The inhibition potential of novel protein was determined for $\mathrm{CYP}_{3} \mathrm{~A} 4, \mathrm{CYP} 2 \mathrm{C}$, CYP2D6, CYP1A2 \& CYP2C19 using Cytochrome P450 inhibitor screening kit. None of these enzyme get inhibited in the presence of molecule.

\section{Biography}

Dr. Sudeep Kumar did his Post Doc in Biotech from Valencia, Spain. He has more than 20 years of experience in Biopharma. He has worked from R\&D to tech transfer and manufacturing of different recombinant proteins from bacteria and mammalian cell culture. He is dealing with different regulatory agencies for approval and faced WHO, USFDA and other regulatory audits. He also actively involved with clinical research team for Preclinical and clinical trials of recombinant proteins and vaccine. He established the VLP technology platform for different vaccine in India in collaboration with Novavax. At present his major research area to develop new recombinant protein for different cancer. 


\title{
10. Relevance of FoxO3a in tamoxifen resistant breast cancer
}

Catia Morelli ${ }^{1}$, Marco Fiorillo ${ }^{2}$, Pietro Rizza ${ }^{1}$, Michele Pellegrino ${ }^{1}$, Alessandra Nigro ${ }^{1}$, Elena Ricci ${ }^{1}$, Marilena Lanzino $^{1}$, Michael Lisanti ${ }^{2}$, Sebastiano Andò ${ }^{1}$, Diego Sisci ${ }^{1}$

\author{
${ }^{1}$ Department of Pharmacy and Health and Nutritional Sciences, University of Calabria, Rende, Cosenza \\ 87036, Italy. \\ ${ }^{2}$ Translational Medicine, School of Environment and Life Sciences, Biomedical Research Centre (BRC), \\ University of Salford, Greater Manchester M5 4WT, UK.
}

Resistance to endocrine treatments is a major clinical challenge in the management of estrogen receptor positive $(\mathrm{ER}+)$ breast cancers $(\mathrm{BC})$. Although multiple mechanisms leading to endocrine resistance have been proposed, the poor outcome of this subgroup of BC patients demands additional studies. Here we show that the expression of FoxO3a transcription factor is reduced in ER+ BC cells that developed resistance to Tamoxifen (Tam), TamR cells, due to its hyper-phosphorylation and degradation mediated by a hyperactive ERK1/2 pathway. On the other hand, FoxO3a silencing counteracts Tam induced growth inhibition in wtMCF-7, demonstrating that FoxO3a is a mediator of cell response to Tam.

FoxO3a re-activation in TamR/FoxO3a inducible clones, developed to express a constitutively active FoxO3a under tetracyclines control, re-established the sensitivity to the antiestrogen, inhibiting proliferation and cell cycle progression, as well as restoring Tam dependent apoptotic response.

Proteomics analysis unveiled novel interesting and potential mediators of the anti-proliferative and proapoptotic activity of the transcription factor, while Kaplan-Meier survival curves showed that high levels of FoxO3a transcripts strongly correlate to a positive response to Tam therapy in BC patients. Finally, FoxO3a induction by the anti-convulsant Lamotrigine (LTG), strongly reduced tumor mass in TamR-derived mouse xenografts.

Altogether, our data indicate that $\mathrm{FoxO} 3 \mathrm{a}$ is a good prognostic factor in $\mathrm{ER}+\mathrm{BC}$, predicting a positive response to endocrine therapy, and a key target to be exploited in combination therapy. In this context, LTG might represent a valid candidate to be used as an adjuvant to Tam therapy in patients at risk.

\section{Biography}

Catia Morelli has completed her PhD from University of Calabria, Cosenza, Italy, after a 4 years training period (1999-2003) at the Kimmel Cancer Center, Thomas Jefferson University, Philadelphia, PA (USA). She is now Researcher at the Department of Pharmacy and Health and Nutritional Sciences at the University of Calabria, recently awaded as Department of Excellence by the Italian Ministry of Education, University and Research. She encounters more than 35 papers in reputed journals and she is also cofounder of NanoSiliCal Devices, a spin-off company of the University of Calabria, developing silica-based nanosystems to be employed in targeted therapy (http://www.nanosilicaldevices.com/en/).

\section{Deciphering human $B$ cell repertoire with next-generation sequencing technology}

\author{
DuckKyun Yoo ${ }^{1}$, Wonjun Yang ${ }^{1}$, Kihyun Kim ${ }^{1}$, Sangil Kim ${ }^{1}$, Sung Min Kim ${ }^{1}$, Hyori Kim ${ }^{2}$, Jinhee Kim ${ }^{3}$, So \\ Young Chang ${ }^{3}$, Meehyun Ko ${ }^{3}$, Ji-Young Min ${ }^{3}$, Haejun Han ${ }^{4}$, Taehoon Ryu ${ }^{4}$, Jungeun Kim ${ }^{4}$, Junho Chung ${ }^{1,5,6}$ \\ ${ }^{1}$ Seoul National University College of Medicine, Seoul 03080, South Korea. \\ ${ }^{2}$ Asan Institute for Life Sciences, Asan Medical Center, Seoul 05505, South Korea.
}


${ }^{3}$ Institut Pasteur Korea, Gyeonggi-Do 464-400, South Korea.

${ }^{4}$ Celemics, Inc., Geumcheon-gu, Seoul 08506, South Korea.

${ }^{5}$ Samsung Biologics, Incheon 21987, South Korea.

${ }^{6}$ GSK Vaccines Research \& Development, Rockville MD 20850, USA.

Next-generation sequencing (NGS) has allowed a massive increase in capacity to sequence genomes at relatively low cost and in a short time frame. It has revolutionized multiple aspects of biological research and is actively being adopted into profiling human B cell receptor (BCR) repertoires. Several NGS platforms are currently available, with average read lengths of $75 \mathrm{bp}$ to $8,500 \mathrm{bp}$ and different error rates.

Using NGS, we successfully constructed database of human BCR repertoire from convalescent patients who recovered from severe fever with thrombocytopenia and middle east respiratory syndrome. Afterwards, we developed algorithms for analyzing the diversity, enrichment pattern, accumulation of somatic hyper-mutation in BCR repertoire. Through in silico analysis we selected clones of interest and prepared recombinant antibodies using a mammalian transient expression system. Their reactivity to viral coat proteins and virus-neutralizing capability was confirmed in in vitro and in vivo experiments.

Currently we are investigating the clinical value of BCR repertoire profiling in autoimmune disease patients including Neuromyelitis Optica and various cancers.

\section{Biography}

Junho Chung has completed his MD, PhD from Seoul National Univ. College of Medicine, South Korea. He is the professor of Seoul National Univ., in the Department for Biochemistry and Molecular Biology. He has published more than 95 papers in reputed journals and has been serving as a vice-editor-in chief of Journal of Cancer Research and Clinical Oncology.

\section{Biobank in Pilsen, Czech Republic, efficient tool for cancer research and personalised treatment}

\section{Judita Kinkorová, Ondřej Topolčan}

Department of Immunochemistry, University Hospital Pilsen, Pilsen 304 60, Czech Republic.

Biobank in University Hospital (UH) Pilsen is newly established (opened in April 2017) hospital integrated biobank, based on previous high quality sample collection. Sinece 2000 the hospital respository started to collect and store systematically biological material of patiens for research in the UH. The main diagnoses for sample collection are oncological samples of breast cancer, colorectal cancer, liver and kidney carcinoma, and prostate cancer.

To be a fully-fledged biobank the following activites have been realised: collection and storage high quilty biological material (blood, serum and plasma) of oncological patients, collection management and storage of related data, implementation of ethical, social and legal issues, harmonization of IT systems, implementation of relevant requrements for providing biological material to the Czech Republic and abroad, preparation of education and training programmes and courses, active participation on BBMRI ERIC activities, and cross-boarder collaboration in the BRoTHER projet.

Currently is the biobank ready to implement aspects of personalised medicine in practice. Regarding the high quality aspects the selected long and short term stability of tumor markers are studied. Together 
with the standard diagnostic procedures like biomarkers, imaging techniques, and clinical stage (multidisciplinary approach), new diagnostic algorithms based on multiparametric and multidisciplinary approach for selected diagnoses are created and brings benefits both for the patient and the hospital.

Personalized medicine approach in University Hospital is closely connected with the education programme realised at Faculty of Medicine in Pilsen Charles University.

\section{Biography}

Judita Kinkorová has completed his PhD from Academy of Science of the Czech Republic in Prague. She is the associate professor, in the Department for immunochemistry University Hospital Pilsen and a manager of biobank in University Hospital Pilsen. She has published more than 40 papers in reputed journals and has been serving as an editorial board member of EPMA Journal.

\section{TM4SF5-mediated hepatic carcinogenesis}

\section{Jung Weon Lee}

Department of Pharmacy, College of Pharmacy, Seoul National University, Seoul 08826, Korea.

Diverse functions of cancer cells including proliferation, migration and invasion critically and greatly depend on extracellular environment during their survival and metastasis. The environment consists of extracellular matrix proteins, neighboring cells, and soluble factors, including of cytokines, chemokines, and growth factors. Efficient dissemination of a cancer cell from a primary tumor mass and invasion through the environment can result in a successful metastasis. Therefore, identification of biological cancer marker(s) playing roles in diverse cancerous functions would be beneficial for clinical and therapeutic purposes, and can be performed in $2 \mathrm{D}$ flat, $3 \mathrm{D}$ cell and tissue cultures, and animal models. Transmembrane $4 \mathrm{~L}$ six family member 5 (TM4SF5) is a transmembrane glycoprotein of the transmembrane $4 \mathrm{~L}$ six family, a branch of the tetraspan(in) family, highly expressed in many types of cancers including hepatic cancer, and shown to cause epithelial-mesenchymal transition. TM4SF5 in hepatocytes is induced by TGF $\beta 1$-mediated Smad and EGFR activation and involved in liver fibrosis. Transgenic animal showed that overexpression of TM4SF5 in liver caused inflammatory, fatty, and fibrotic phenotypes, indicating that TM4SF5 expression following actions by TGF $\beta 1$ prevalent in chronic liver injury situation may lead to playing regulatory roles in functions for different metabolic pathways. Further, disease-prone TM4SF5 transgenic mice showed enhanced expressions of CD34, HIF1-a, AFU, and AFP in the livers. A small calchone compound, TSAHC and chimeric anti-TM4SF5 antibody could successfully block the TM4SF5-mediated regulation of metabolic activities, fibrosis, and xenograft tumor formation.

\section{Biography}

Dr. Jung Weon Lee is a Professor at Dept. of Pharmacy, Seoul National University (SNU), Korea. He got Ph.D. in Pharmacology, University of North Carolina at Chapel Hill, NC, USA. He had a postdoc period at Memorial Sloan-Kettering Cancer Center, NY, USA. In 2001, He came back to Korea, and in 2009 moved to Dept. of Pharmacy, SNU. His researches focuse on how cellular functions occur at the molecular levels. His researches focuses on the roles of a tetraspanin, $\mathrm{TM} 4 \mathrm{SF} 5$, in metabolic dysorders, fibrosis, tumorigenesis and metastasis, and on the anti-TM4SF5 reagents to block TM4SF5-mediated diseases (Lab homepage: http://www.snupharm.ac.kr/jwl/). 


\title{
14. The histone methyl transferase DOT1L is a key functional partner of estrogen receptor alpha for regulation of gene transcription in hormone-responsive breast cancer
}

\author{
Giovanni Nassa ${ }^{1,2}$, Annamaria Salvati ${ }^{1}$, Giorgio Giurato ${ }^{1,2}$, Roberta Tarallo', Francesca Rizzo ${ }^{1}$, Lorenza \\ Mautone ${ }^{1}$, Domenico Rocco ${ }^{1}$, Tuula Anneli Nyman ${ }^{3}$, Alessandro Weisz ${ }^{1}$ \\ ${ }^{1}$ Laboratory of Molecular Medicine and Genomics, Department of Medicine, Surgery and Dentistry "Scuola \\ Medica Salernitana", University of Salerno, Baronissi, SA 84084, Italy. \\ ${ }^{2}$ Genomix4Life S.r.l., University of Salerno, Baronissi, SA 84084, Italy. \\ ${ }^{3}$ Proteomics Core Facility, Institute of Clinical Medicine, University of Oslo, Oslo 0315, Norway.
}

Estrogen Receptor alpha $(\mathrm{ER} \alpha)$ is a key mediator of estrogen signaling in hormone-responsive breast cancer (BC) and target of endocrine therapies, where resistance to treatment is a major problem in clinical management of these patiens. A better understanding of the molecular mechanisms of ER $\alpha$ actions in cancer may lead to identification of new therapeutic targets against endocrine terapy-resistant tumors. We recently identified the epigenetic writer DOT1L (DOT1 Like Histone Lysine Methyltransferase) as novel a nuclear partner of $\mathrm{ER} \alpha$ in hormone-responsive $\mathrm{BC}$ cells. To investigate the functional role of this enzyme in mediating receptor actions in target cell nuclei, physical and functional ER $\alpha$-DOT1L interaction on chromatin was mapped by Chromatin Immunoprecipitation coupled to Mass Spectrometry (ChIP-MS) and Sequencing (ChIP-Seq), transcriptome profiling (RNA-Seq) coupled to gene silencing and pharmacological inhibition of either protein. Cellular and functional assays were also used to evaluate the impact of the ER $\alpha$-DOT1L complex in hormone-responsive BC cell functions. ChIP-MS confirmed co-recruitment of the two factors on chromatin in a multiprotein complex. Gene expression profiling and Nascent RNA-Seq before and after DOT1L pharmacological inhibition showed that this enzyme is involved in ER $\alpha$-mediated transcriptional regulation of several estrogen responsive genes, including ER $\alpha$ itself. ChIP-Seq mapped co-occupancy of several chromatin sites by both proteins, including the ER $\alpha$ gene promoter itself. These results reveal that physical and functional association between ER $\alpha$ and DOT1L is a key molecular event in hormonal control of $\mathrm{BC}$ cell functions, suggesting that this enzyme might represent a potential drug target against hormone-responsive tumors.

\section{Biography}

Dr. Giovanni Nassa completed his PhD at University of Campania "Luigi Vanvitelli" and presently is fixedterm Researcher (RTDa) of General Pathology at the Department of Medicine, Surgery and Dentistry "SMS" of the University of Salerno. AIRC Fellow during the early stages of his career, he was also visiting scientist and FEBS fellow at the Institute of Biotechnology and Biomedicum of the University of Helsinki. He published more than 30 papers in international peer-review journals, with research mainly focusing on the epigenetic mechanisms underlying hormone-responsive breast cancer pathogenesis and loss of response to endocrine therapy during progression.

\section{IL-4 as a potential treatment for cancer-induced muscle wasting}

\section{Paola Costelli}

Department of Clinical and Biological Sciences, University of Turin, Turin 10126, Italy.

Skeletal muscle wasting, one of the most relevant features of cancer cachexia, is mainly associated with marked alterations of protein turnover. Few years ago the observation that also myogenesis is impaired in 
cancer hosts was reported. In particular, Pax7+ cells accumulate in the muscle of tumor-bearing animals without proceeding into differentiation and fusing with existing myofibers. Such impairment was proposed to depend on persistently expressed Pax7 due to NF- $\mathrm{BB}$ hyperactivation. The reduced myogenesis does not depend on an intrinsic defect of muscle stem cells, since they can easily differentiate in vitro and can fuse with damaged myofibers in an experimental model of muscle dystrophy. However, myogenic precursors isolated from mice bearing the $\mathrm{C} 26$ tumor cultured in proliferation medium spontaneously differentiate to adipocytes.

To investigate the mechanisms underlying such "adipogenic drive", mice bearing the C26 carcinoma were treated intraperitoneally with IL-4, a cytokine previously shown to inhibit adipogenesis in muscle. The results obtained show that muscle wasting was partially prevented in tumor-bearing animals receiving IL4 , in terms of both tissue weight and myofiber cross sectional area. The improved muscle phenotype is associated with reduced levels of phosphorylated ERK, in agreement with previous data. The spontaneous adipogenic differentiation is markedly reduced in primary cultures of myogenic precursors isolated from the $\mathrm{C} 26$ hosts treated with IL-4 in comparison to those obtained by untreated animals. This observation suggested that IL-4, by inhibiting adipogenesis, could force myogenesis. Consistently, when IL-4 is administered to tumor-bearing animals in which muscle damage was induced by injection of $\mathrm{BaCl}$, regeneration occurred faster than in the untreated $\mathrm{C} 26$ hosts.

On the whole these results suggest that primary cultures of myogenic precursors isolated from tumorbearing animals are committed to differentiate to adipocytes, but can be forced through myogenesis by adequate stimuli. By contrast, the muscle microenvironment existing in vivo in the tumor host is able to inhibit both myogenic and adipogenic differentiation of these precursors, that indeed accumulate in the tissue. Preliminary data suggest that the lack of IL-4 production could play a role in this regard.

\section{Lenvatinib in anaplastic thyroid cancer}

\section{Alessandro Antonelli ${ }^{1}$, Silvia Martina Ferrari ${ }^{1}$, Ilaria Ruffilli ${ }^{1}$, Giusy Elia ${ }^{1}$, Francesca Ragusa ${ }^{1}$, Sabrina Rosaria Paparo', Claudia Caruso', Poupak Fallahi}

${ }^{1}$ Department of Clinical and Experimental Medicine, University of Pisa, Pisa 56126, Italy.

${ }^{2}$ Department of Translational Research and New Technologies in Medicine and Surgery, University of Pisa, Pisa 56126, Italy.

The oral multitargeted tyrosine kinase inhibitor lenvatinib acts against VEGFR1-VEGFR3, FGFR1-FGFR4, PDGFR $\alpha$, RET and v-kit Hardy-Zuckerman 4 feline sarcoma viral oncogene homolog (KIT) signaling networks involved in tumor angiogenesis.

The antitumor activity of lenvatinib $(1 \mathrm{nmol} / \mathrm{L}, 100 \mathrm{nmol} / \mathrm{L}, 1 \mathrm{mcmol} / \mathrm{L}, 10 \mathrm{mcmol} / \mathrm{L}, 25 \mathrm{mcmol} / \mathrm{L}$ and $50 \mathrm{mcmol} / \mathrm{L}$ ) has been investigated in primary anaplastic thyroid cancer (ATC) cells, in the human cell line $8305 \mathrm{C}$ (undifferentiated thyroid cancer) and in an ATC-cell line (AF), in vitro; and in vivo in AF cells injected in $\mathrm{CD}$ nu/nu mice.

Lenvatinib significantly reduced ATC cell proliferation $(P<0.01$, ANOVA), increasing apoptosis $(P<$ 0.001 , ANOVA). Furthermore, lenvatinib inhibited migration $(P<0.01)$ and invasion $(P<0.001)$ in ATC, inhibited EGFR, AKT and ERK1/2 phosphorylation and down-regulated cyclin D1 in ATC cells. Moreover, lenvatinib significantly inhibited $8305 \mathrm{C}$ and $\mathrm{AF}$ cell proliferation, increasing apoptosis. 
AF cells were injected subcutaneously into $\mathrm{CD}$ nu/nu mice and tumor masses were evident after 20 days. Tumor growth was significantly inhibited by lenvatinib $(25 \mathrm{mg} / \mathrm{kg} / \mathrm{day})$, as the VEGF-A expression and microvessel density in AF tumor tissues.

In conclusion, we show for the first time the antitumoral effect of lenvatinib in primary human ATC cell cultures obtained from patients. These results could open the way to the clinical use of lenvatinib in the treatment of patients with ATC.

\section{Biography}

Prof. Antonelli is currently Director of the Immuno-Endocrine Section of Internal Medicine and Associate Professor in the Department of Clinical and Experimental Medicine, University of Pisa, Azienda Ospedaliera-Universitaria Pisana, Pisa, Italy.

He graduated in Medicine cum laude at the University of Pisa in 1982, where he received the post-graduate Specialization in Endocrinology in 1985 and in Occupational Health in 1987. He received also the postgraduate Specialization in Oncology (at the University La Sapienza, Rome, Italy) in 1992.

His researches have been published in more than 320 articles on International journals (Impact Factor > 1200; H index 60).

\section{Novel inducers of noncanonical and immunogenic cell death}

\section{Marc Diederich}

Department of Pharmacy, College of Pharmacy, Seoul National University, Seoul 151742, South Korea.

Apoptosis was most often considered as the most important type of cell death and numerous compounds triggering this programmed form of cell demise were explored. Over the last 10 years, other cell death modalities were also described, and proof of concept was provided that such compounds are efficiently able to target cancer cells that already became resistant to conventional apoptosis inducers. Moreover, the interplay between different cell death modalities gained interest ${ }^{[1]}$. We became interested to assess the anticancer effect of compounds that were shown to kill cells by triggering various necrosis-related phenotypes $^{[2,3]}$. We believe that these compounds provide a more efficient therapeutic outcome. For our research we essentially investigated compounds of natural origins from both marine ${ }^{[4-6]}$ and terrestrial sources $^{[7-10]}$. Multiple forms of regulated necrosis and the in-depth elucidation of the corresponding cell signaling pathways allowed to better predict the immunogenic potential of cancer cells dying by noncanonical cell death mechanisms ${ }^{[11-13]}$. This presentation will cover the effect of various natural and hemisynthetic compounds while describing their cell death-inducing activity leading to secretion or exposure of immunogenic cell death markers ${ }^{[14-16]}$.

\section{REFERENCES}

1. Radogna F, Dicato M, Diederich M. Cancer-type-specific crosstalk between autophagy, necroptosis and apoptosis as a pharmacological target. Biochem Pharmacol 2015;94:1-11.

2. Florean C, Song S, Dicato M, Diederich M. Redox biology of regulated cell death in cancer: a focus on necroptosis and ferroptosis. Free Radic Biol Med 2019;134:77-189.

3. Diederich M, Cerella C. Non-canonical programmed cell death mechanisms triggered by natural compounds. Semin Cancer Biol 2016;40-41:4-34.

4. Lee JY, Orlikova B, Diederich M. Signal transducers and activators of transcription (STAT) regulatory networks in marine organisms: from physiological observations towards marine drug discovery. Mar Drugs 2015;13:4967-84. 
5. Schnekenburger M, Dicato M, Diederich M. Epigenetic modulators from "The Big Blue": a treasure to fight against cancer. Cancer Lett 2014;351:182-97.

6. Schumacher M, Kelkel M, Dicato M, Diederich M. Gold from the sea: marine compounds as inhibitors of the hallmarks of cancer. Biotechnol Adv 2011;29:531-47.

7. Cerella C, Teiten MH, Radogna F, Dicato M, Diederich M. From nature to bedside: pro-survival and cell death mechanisms as therapeutic targets in cancer treatment. Biotechnol Adv 2014;32:1111-22.

8. Mazumder A, Cerella C, Diederich M. Natural scaffolds in anticancer therapy and precision medicine. Biotechnol Adv 2018;36:1563-85.

9. Morceau F, Chateauvieux S, Orsini M, Trecul A, Dicato M, et al. Natural compounds and pharmaceuticals reprogram leukemia cell differentiation pathways. Biotechnol Adv 2015;33:785-97.

10. Schnekenburger M, Dicato M, Diederich M. Plant-derived epigenetic modulators for cancer treatment and prevention. Biotechnol Adv 2014;32:1123-32.

11. Radogna F, Dicato M, Diederich M. Natural modulators of the hallmarks of immunogenic cell death. Biochem Pharmacol 2019;162:5570 .

12. Radogna F, Diederich M. Stress-induced cellular responses in immunogenic cell death: Implications for cancer immunotherapy. Biochem Pharmacol 2018;153:12-23.

13. Diederich M, Muller F, Cerella C. Cardiac glycosides: from molecular targets to immunogenic cell death. Biochem Pharmacol 2017;125:1-11.

14. Mazumder A, Lee JY, Talhi O, Cerella C, Chateauvieux S, et al. Hydroxycoumarin OT-55 kills CML cells alone or in synergy with imatinib or Synribo: involvement of ER stress and DAMP release. Cancer Lett 2018;438:197-218.

15. Lee JY, Talhi O, Jang D, Cerella C, Gaigneaux A, et al. Cytostatic hydroxycoumarin OT52 induces ER/Golgi stress and STAT3 inhibition triggering non-canonical cell death and synergy with BH3 mimetics in lung cancer. Cancer Lett 2018;416:94-108.

16. Ji S, Lee JY, Schror J, Mazumder A, Jang DM, et al. The dialkyl resorcinol stemphol disrupts calcium homeostasis to trigger programmed immunogenic necrosis in cancer. Cancer Lett 2018;416:109-23.

\section{Biography}

Marc Diederich earned his $\mathrm{PhD}$ in molecular pharmacology in 1994 from the University Henri Poincaré Nancy 1, France. After training at the University of Cincinnati, USA, he focused his research on cancer and leukemia cell signaling pathways and gene expression mechanisms triggered by natural compounds with epigenetic-, anti-inflammatory- and cell death-inducing potential. He directs the Laboratory for molecular and cellular biology of cancer (LBMCC) at Kirchberg Hospital in Luxemburg. He was appointed associate Professor of Biochemistry at the College of Pharmacy of Seoul National University in 2012. In 2017, he was tenured and promoted to full professor at SNU.

\section{Co-expression of immune checkpoint genes at the cancer/antigen-presenting-cell side of the immunological synapse}

\section{Raya Leibowitz-Amit ${ }^{1}$, Amir Herman ${ }^{2}$, Adi Layani ${ }^{1}$, Paula Dobosz $^{1}$, Yehezkel Sidi', Dror Avni ${ }^{1}$ \\ ${ }^{1}$ Oncology institute and cancer research center, Sheba medical center, Tel-Hashomer 52621, Israel. \\ ${ }^{2}$ Orthopedics department, Assuta medical center, Ashdod, Tel Aviv 69710, Israel.}

Introduction: The interface between T lymphocytes and cancer cells or antigen-presenting cells (APC), termed "the immunological synapse" (IS), comprises of both co-inhibitory and co-stimulatory checkpoint proteins that modulate the signal transmitted to $\mathrm{T}$ lymphocytes, leading to either activation, anergy or exhaustion. Monoclonal antibodies against checkpoint proteins (designated "checkpoint inhibitors") have anti-neoplastic activity in several malignancies, but not all cancers and not all patients within a given cancer respond.

Methods: We analysed the tumor cancer genome atlas database for the expression of 15 checkpoint mRNAs suggested from the literature to be expressed at the cancer/APC side of the IS in 28 tumor types. The Spearman rho correlation co-efficient for the co-expression of each pair of checkpoint mRNAs was 
calculated. For each cancer type, we calculated the average correlation co-efficient of any combination of 2-15 mRNAs.

Results: The number of co-expressed checkpoint genes with an average Spearman rho co-efficient of above 0.5 was significantly different between tumor types, ranging from 10 to 0 . Thyroid carcinoma, testicular cancer and melanoma were the three tumors with the highest number of co-expressed checkpoint mRNAs, whereas in acute leukemia there were no co-expressed checkpoint mRNAs, as predicted. Similarly, the nature and mix of co-stimulatory and co-inhibitory checkpoint mRNAs was different between different tumor types, with each tumor exhibiting a different "network" of checkpoint co-expression with differing strengths of correlations.

Conclusion: Our bioinformatic analysis indicates that both co-inhibitory and co-stimulatory checkpoint mRNAs are co-expressed in solid malignancies in varying extents in different cancers. We propose that the differences in these "checkpoint networks" formed on the cancer side of the IS take part in determining cancer immunogemicity. It is tempting to speculate that these networks may also take part in determining the potential of a tumor type to respond to the currently-available immune checkpoint inhibitors.

\title{
Biography
}

Raya Leibowitz completed her PhD and her MD at the Tel-Aviv university, Israel. She is currently a senior medical oncologist (clinician-investigator) at the Sheba medical center and an assistant professor at the department of oncology, faculty of medicine, Tel-Aviv university. Her major scientific work until now (first and last authorship) was published in Nature Medicine, Cancer Research, Annals of oncology and Molecular Cancer. Among other grants, she was a recipient of the "melanoma research alliance young investigator award", the "Gerald Kirsh" humanitarian award and the "Conquer Cancer foundation" merit award twice.

\section{Sunitinib in thyroid cancer: a review of the literature}

\author{
Giusy Elia', Silvia Martina Ferrari ${ }^{1}$, Ilaria Ruffilli', Francesca Ragusa', Sabrina Rosaria Paparo', Claudia \\ Caruso ${ }^{1}$, Poupak Fallahi ${ }^{2}$, Alessandro Antonelli ${ }^{1}$ \\ ${ }^{1}$ Department of Clinical and Experimental Medicine, University of Pisa, Pisa 56126, Italy. \\ ${ }^{2}$ Department of Translational Research and New Technologies in Medicine and Surgery, University of Pisa, \\ Pisa 56126, Italy.
}

Sunitinib is a multi-targeted tyrosine kinase inhibitor (TKI), acting on different receptors for plateletderived growth factor receptors and vascular endothelial growth factor receptors, c-KIT, fms-related tyrosine kinase 3 and RET, leading to tumor vascularization reduction, tumor cell apoptosis, and causing tumor shrinkage.

It has been approved for the treatment of pancreatic neuroendocrine tumors, imatinib-resistant gastrointestinal stromal tumor and renal carcinoma.

In vitro studies have been conducted on the RET/PTC1 cell, showing that sunitinib is able to target the cytosolic MEK/ERK and SAPK/JNK pathways inhibiting cell proliferation and causing stimulation of sodium/iodide symporter gene expression.

Also in vitro and in vivo studies conducted on anaplastic thyroid cancer cells have shown the efficacy of sunitinib, also as first- and second-line TKI therapy in patients with advanced dedifferentiated thyroid cancer, or medullary thyroid cancer. 
The most frequent adverse events are fatigue, decreases in blood cell counts (especially leukocytes), diarrhea, hand-foot skin reaction, nausea, hypertension and musculoskeletal pain. A dose of $37.5 \mathrm{mg} / \mathrm{day}$ sunitinib is well-tolerated, and effective.

To sum up, although sunitinib is promising in the therapy of differentiated thyroid carcinoma, until now no phase III studies have been published. Further prospective researches are needed to evaluate the real effectiveness of sunitinib in aggressive thyroid cancer.

\section{Biography}

Giusy Elia is graduated in Biology applied to Biomedicine in 2012 and specialized in Clinical Pathology and Clinical Biochemistry in 2017 at the University of Pisa (Italy). Her areas of interests range from Immunology, Pharmacology to Biochemistry. Her researches have been published on International journals $(\mathrm{HI}=5)$.

\section{Vandetanib in anaplastic thyroid cancer}

\section{Silvia Martina Ferrari ${ }^{1}$, Poupak Fallahi ${ }^{2}$, Ilaria Ruffilli', Giusy Elia ${ }^{1}$, Francesca Ragusa ${ }^{1}$, Sabrina Rosaria Paparo ${ }^{1}$, Claudia Caruso ${ }^{1}$, Alessandro Antonelli ${ }^{1}$}

${ }^{1}$ Department of Clinical and Experimental Medicine, University of Pisa, Pisa 56126, Italy.

${ }^{2}$ Department of Translational Research and New Technologies in Medicine and Surgery, University of Pisa, Pisa 56126, Italy.

Anaplastic thyroid cancer (ATC) represents about $1 \%$ of thyroid cancer (TC), and it is one of the most aggressive human tumors, representing approximately $15 \%-40 \%$ of TC deaths. The multimodal treatment, that includes debulking, chemotherapy, and hyperfractionated accelerated external beam radiotherapy, is the most effective treatment, with a median survival of 10 months. New drugs have been recently evaluated in ATC, but until now no significant improvement of survival has been observed.

Vandetanib is an oral once-daily multi-tyrosine kinase inhibitor, inhibiting the activation of RET, EGFR, VEGFR-2, VEGFR-3, and slightly VEGFR-1, and with a potent anti-angiogenic activity.

Its antitumor activity was tested at different concentrations $(1 \mathrm{nmol} / \mathrm{L}, 100 \mathrm{nmol} / \mathrm{L}, 1 \mu \mathrm{mol} / \mathrm{L}, 10 \mu \mathrm{mol} / \mathrm{L}$, $25 \mu \mathrm{mol} / \mathrm{L}$ and $50 \mu \mathrm{mol} / \mathrm{L}$ ) in primary ATC cells, in the $8305 \mathrm{C}$ continuous cell line, and in AF cells; and in $8305 \mathrm{C}$ cells in $\mathrm{CD}$ nu/nu mice.

Vandetanib decreased significantly ATC cells proliferation $(P<0.01$, ANOVA), inducing apoptosis dosedependently $(P<0.001$, ANOVA), and inhibiting migration $(P<0.01)$ and invasion $(P<0.001)$. Vandetanib inhibited EGFR, AKT and ERK1/2 phosphorylation and down-regulated cyclin D1 in ATC cells.

In $8305 \mathrm{C}$ and $\mathrm{AF}$ cells, vandetanib inhibited significantly the proliferation, inducing also apoptosis. $8305 \mathrm{C}$ cells were injected sc in CD nu/nu mice and tumor masses became detectable 30 days after. Vandetanib (25 $\mathrm{mg} / \mathrm{kg} / \mathrm{die}$ ) inhibited significantly tumor growth and VEGF-A expression and microvessel density in $8305 \mathrm{C}$ tumor tissues.

In conclusion, we show the antitumor and antiangiogenic activity of vandetanib in ATC, paving the way to a future clinical evaluation. 


\title{
Biography
}

Silvia Martina Ferrari is graduated in Biological Sciences cum laude in 2002 and specialized in Clinical Pathology in 2007 at the University of Pisa (Italy). Her principal areas of expertise are autoimmune thyroid disorders, chemokines and cytokines, type 1 diabetes, systemic autoimmune disorders, HCV-associated thyroid disorders and thyroid cancer. Her researches have been published in more than 158 articles on International journals $(\mathrm{HI}=38)$. She serves as an editorial board member and is Referee and Reviewer of many scientific International journals.

\section{Mitochondrial dynamics and chemoresistance in ovarian cancer}

\author{
Youngjin Han ${ }^{1}$, Yong Sang Song ${ }^{1,2}$ \\ ${ }^{1}$ Cancer Research Institute, Seoul National University, Seoul 03080, South Korea. \\ ${ }^{2}$ Department of Obstetrics and Gynecology, College of Medicine, Seoul National University, Seoul 03080, \\ South Korea.
}

Various cellular and acellular components constitute tumor microenvironment affecting metabolism and malignant phenotypes of cancer. Hypoxia and increased level of reactive oxygen species (ROS) are frequently observed in many types of malignant tissues composed of cancer cells and surrounding tumor microenvironment, including ovarian cancer. In response to the increased level of ROS, cancer cells activate antioxidant mechanisms to counterbalance increased ROS. PGC1 $\alpha$ is a key molecule critical for mitochondrial biogenesis and upregulation of antioxidant enzymes. In our previous research, we reported that PGC1 $\alpha$ is associated with cisplatin-resistance in tumor spheres. Tumor sphere cells acquired stem cell-like properties together with increased production of ROS. ROS was shown to be important for maintaining stemness and PGC1 $\alpha$ expression of the tumor sphere cells in ovarian cancer. Decreased sensitivity to cisplatin was observed in both tumor sphere and PGC1 $\alpha$-overexpressing cells, while silencing PGC1 $\alpha$ sensitized the tumor sphere cells to cisplatin. Along with upregulation of PGC1 $\alpha$ expression in tumor sphere cells, mitochondrial fission was increased in comparison to their parental cells. Similar to the in-vivo environment of the malignant tumor, tumor spheres exhibit hypoxia at the core. The center of tumor spheres is exposed to hypoxia like in vivo tumor. As the tumor sphere grows larger in size, a hypoxic gradient towards the core of the sphere is created. Next, we investigated the effect of hypoxia on mitochondrial dynamics. Mitochondria, dynamic intracellular organelles, go through incessant processes of fission/fusion and biogenesis by various stimuli in tumor microenvironment. Under hypoxic $(<1 \% \mathrm{O} 2)$ culture conditions, mitochondrial fission was increased together with increased resistance to cisplatin in ovarian cancer cells. Inhibition of mitochondrial fission enhanced sensitivity of cancer cells to cisplatin. Our findings suggest that PGC1 $\alpha$-induced mitochondrial biogenesis and alteration of mitochondrial dynamics by hypoxia could cause the cisplatin resistance of ovarian cancer cells. The changes in mitochondrial biogenesis and dynamics of cancer cells adapted to tumor microenvironment could be a novel therapeutic target to overcome chemoresistance in ovarian cancer.

\section{Biography}

Prof. Yong Sang Song is with the Department of Obstetrics and Gynecology, College of Medicine, Seoul National University, Korea. He received MD, College of Medicine, Seoul National University, Korea from 1977 to 1983; the MS and the PhD, Postgraduate School Seoul National University, Korea. His major interests are molecular mechanisms of tumors, especially the role of tumor microenvironment in cancer cell metabolism and chemoresistance and precision medicine in ovarian cancer. He is particularly interested in the impacts of components of tumor microenvironment in ovarian cancer progression. He has published more than 300 papers in the science citation index journals. 


\title{
22. A strategy for the prevention of thyroid cancer with a focus on the experience of managing radio-induced thyroid cancer after the Chernobyl Accident
}

\author{
Valentina Drozd ${ }^{1,2}$, Daniel Igor Branovan ${ }^{1}$, Michael Fridman $^{3}$, Nikolay Shiglik $^{1}$, Sergey Vershenya ${ }^{2}$, Igor \\ Makarov $^{2}$, Maxim Lushik ${ }^{4}$, Larisa Danilova ${ }^{4}$ \\ ${ }^{1}$ New York Ear, Nose and Throat Institute, New York, NY 10003, United States. \\ ${ }^{2}$ LTD MedEx-lab, Minsk 220013, Belarus. \\ ${ }^{3}$ Republican Centre for Thyroid Tumors, Minsk 220013, Belarus. \\ ${ }^{4}$ Belorussian medical academy for postgraduate education, Minsk 220013, Belarus.
}

One of the lessons learned from Chernobyl is the dramatic increase in incidence of thyroid cancer in Belarus. However the incidence of thyroid cancer has been steadily increasing during the last 30 years worldwide most markedly in the Republic of Korea, France, Italy, Australia, and USA. Analyzing this problem, some scientists have recently identified this epidemic of thyroid cancer as "overdiagnosis" and "overtreatment". On the other hand, overall incidence-based mortality for thyroid cancer in USA from 1994 to 2013 substantially increased ( $1.1 \%$ per year), and it increased even faster for patients diagnosed with advanced-stage papillary thyroid cancer (2.9\% per year). These findings are consistent with a true increase in the occurrence of thyroid cancer. The main factors contributing to this increase continue to be debated. The experience of managing radio-induced thyroid cancer after the Chernobyl Accident has shown that radiation exposure and the usage of drinking water with a high concentration of nitrates were significantly associated with high incidence of thyroid cancer. Better understanding of the combined impact mechanism of radiation and nitrates exposure and maybe other endocrine disruptors will give us more opportunities to develop new ways of thyroid cancer preventing.

The rising incidence and optimization of management of nodular goiter are also actual problems for Belarus. Surgery for treating of low-risk thyroid papillary microcarcinomas and nodular goiter might be an overtreatment and it is important to develop a less traumatic modalities. To ablate small thyroid nodules (less than $2 \mathrm{~cm}$ in diameter), we started to use new originally developed low-cost device for bipolar radiofrequency ablation - «Thyrablator» designed as a sterile disposable needle for the fine needle biopsy (20-gauge). The first experience showed a promising efficacy and safety of new device - «Thyrablator» as a minimally invasive treatment of thyroid lesions.

More studies are needed to better verify the causes of the epidemic of thyroid cancer in the world and to concentrate our efforts on the prevention and development of new minimally invasive approaches for the treatment of nodular goiter and early forms of thyroid cancer.

\section{Reducing PD-L1 expression with epigenetic modifiers enhances immunotherapy}

\author{
Tessa Knox ${ }^{1}$, Eva Sahakian², Debarati Banik ${ }^{1}$, Melissa Hadley ${ }^{1}$, Erica Palmer ${ }^{1}$, Jennifer Kim $^{1}$, John Powers $^{2}$, \\ Fengdong Cheng ${ }^{1}$, Vincent Gallub ${ }^{1}$, Sarthak Shah ${ }^{1}$, Alejandro Villagra ${ }^{1}$ \\ ${ }^{1}$ The George Washington University, Washington, DC 20052, USA. \\ ${ }^{2}$ H. Lee Moffitt, Tampa, FL 33612, USA.
}

Histone deacetylases (HDACs), were originally described in a limited context as histone modifiers. New evidence has demonstrated that HDACs are also involved in a diverse range of cellular processes that are 
not restricted to the chromatin environment, such as the regulation of the cell cycle/apoptosis and, more recently, a modulator of immune response. However, much remains unknown about the mechanism of action of HDACs and their roles in the immune-biology of cancer. The non-specific nature of pan-HDAC inhibitors results in a narrow therapeutic window of use, limiting the dose and duration due to toxicity. Our group has focused in one specific HDAC, HDAC6, and shown that both the genetic abrogation and pharmacological inhibition of this HDAC modulates the expression of a variety of immune-regulatory proteins in the tumor microenvironment, including PD-L1, PD-L2, MHC class I, B7-H4 and TRAIL-R1. We have previously demonstrated that both pharmacological inhibition and/or genetic abrogation of HDAC6 plays a critical role in the immune check point blockade by down-regulating the expression of PD-L1 and other check-point modulators such as PD-L2, B7-H4, etc. Moreover, we have also observed that in vivo inhibition of HDAC6 reduces tumor growth in B16 and SM1 murine melanoma models within syngeneic immunocompetent hosts. Additionally, we have found that the combination of low doses of the HDAC6i Nexturastat A and checkpoint immune blockade therapies, including anti-PD-1 and antiCTLA4, result in an important improvement in anti-tumor responses in our murine model as evidenced by the reduction of tumor growth when compared to treatment with individual stand-alone agents and the improved modulation of various immune markers. Our studies have also demonstrated that tumors treated with stand-alone check point inhibitor treatments, specifically anti-PD-1, results in a substantial increase in the production of IFN $\gamma$ and IL-2, resulting in the upregulation of PD-L1 and other critical checkpoints in the immune blockade. Similar levels of IFN $\gamma$ and IL-2 were found in the combination subject groups, however, the levels of PD-L1 and PD-L2 were more comparable to the non-treated group. Overall, our evidence suggests that HDAC6i has the potential for use as an adjuvant in ongoing therapeutic options involving the immune check-point blockade.

\section{Trisenox disrupts MDM2-DAXX-HAUSP complex, degrades MDM2, and activates p53 in acute leukemia cells}

\section{Paul Bernard Tchounwou, Sanjay Kumar}

\section{Cellomics and Toxicogenomics Research Laboratory, NIH/NIMHD-RCMI Center for Environmental Health, College of Science, Engineering and Technology, Jackson State University, Jackson, MS 3921 7, USA.}

Trisenox (TX) has been successfully used in the treatment of both de novo and relapsed acute promyelocytic leukemia (APL) patients. It inhibits APL cells growth efficiently through cell cycle arrest and apoptosis, however exact molecular mechanisms of action poorly understood. In present study, we found a new target of TX action that involves the activation of p53 and p21 expression through association with death domain-associated protein (DAXX), disruption of MDM2-DAXX-HAUSP complex and degradation of MDM2 in APL cells. TX-induced stress signal is transmitted by protein kinase (ATM \& ATR) and phosphorylation of CHK1 \& CHK2 at Ser 345 and Thr68 residues leading to complex disruption and accumulation of p53 in APL cells. TX-induced p53 caused cell cycle arrest by regulating expression of p21, cyclins and cyclin dependent kinases proteins and forcing cells to undergo apoptosis by apoptotic proteins expression modulation, mitochondrial membrane depolarization leading to caspase 3 activation. Our immunoprecipitation studies also showed that the complex molecules were well associated in APL cells, and TX disrupted their associations leading to accumulation of p53. We further studied the functional role of p53 in disruption and expression of complex molecules in p53-knock down APL cells using lentiviral shRNA approach. Taken together, our findings showed that TX activates p53, through association of DAXX, disruption of MDM2-DAXX-HAUSP complex, MDM2 degradation in APL cells leading to cell cycle arrest and apoptosis. This novel target of TX activity may be useful for designing new APL drugs. 


\section{Biography}

Dr. Paul B. Tchounwou is Associate Dean of the CSET, Presidential Distinguished Professor, and Director of the NIH-funded RCMI-Center for Environmental Health. He is an internationally recognized biomedical scientist whose research focuses on the mechanisms of action of xenobiotic compounds and cancer therapeutic drugs. He is author of 228 peer-reviewed publications and 507 scientific presentations. $\mathrm{He}$ is the Editor-in-Chief of Environmental Toxicology (New York, USA) and of International Journal of Environmental Research and Public Health (Basel, Switzerland). He has received several awards for excellence including the 2003 Millennium Award for Research Excellence conferred by the White House (Washington DC).

\section{The potential role of milk's amino acid- and miRNA signaling in the pathogenesis of prostate cancer}

\section{Bodo C. Melnik ${ }^{1}$, Swen Malte John ${ }^{1}$, Pedro Carrera-Bastos ${ }^{2}$, Loren Cordain ${ }^{3}$, Gerd Schmitz ${ }^{4}$}

${ }^{1}$ Department of Dermatology, Environmental Medicine and Health Theory, University of Osnabrück, Osnabrück 49074, Germany.

${ }^{2}$ Center for Primary Health Care Research, Lund University, Lund Se-22100, Sweden.

${ }^{3}$ Department of Health and Exercise Science, Colorado State University, CO 80523, USA.

${ }^{4}$ Institute for Clinical Chemistry and Laboratory Medicine, University Hospital Regensburg, Regensburg 93053, Germany.

Epidemiological studies reported a dose relationship between cow's milk intake and prostate cancer (PCa). Lactose intolerance protects against the development of common cancers and a lower incidence of lactose intolerance in comparison to the general population has been reported in PCa patients. Daily milk consumption during adolescence in comparison to less frequent milk consumption increased the risk of advanced PCa in adulthood by 3.2. An increased risk for PCa has also been observed for patients with a history of severe acne, which is another milk-induced disease, pointing to common underlying signaling pathways. Several components of milk such as dairy protein, calcium, galactose, miRNAs, androgen precursors and estrogens derived from high-yield dairy cows have been implicated to play a role in milk-induced PCa. We provide translational evidence, that milk's essential amino acids via enhancing the secretion of insulin and insulin-like growth factor 1 result in AKT-stimulated mouse double minute 2-mediated degradation of the tumor suppressor p53. Moreover, milk exosomal miRNA-125b, which survives pasteurization, may directly repress TP53 mRNA. Milk-derived miRNA-148a via depletion of DNA methyltransferase 1 and hypomethylation of cancer-germline genes may promote epithelialmesenchymal transition and cancer stem cells. Although boiling and ultraheat treatment of milk eliminates milk's miRNA signaling, milk's amino acid signaling is not affected by thermal procedures. We recommend that patients with severe acne vulgaris, adults with a history of severe acne, as well as individuals with a family history of PCa should refrain from pasteurized cow's milk to prevent PCa.

\section{Biography}

After his medical studies at the University of Münster, Germany, Bodo C. Melnik was visiting scientist at the University of California San Francisco from 1982-1984. From 1984-1990, he was resident at the Department of Dermatology, University of Düsseldorf. He is currently Associate Professor at the Department of Dermatology, Environmental Medicine and Health Theory, University of Osnabrück, Germany, member of the European Society for Dermatological Research and coeditor of the Journal of Translational Research. His research interests are milk signaling and milk microRNA signal transduction in diseases of civilization such as acne and prostate cancer. 


\section{Human-enzyme mediated, systemic depletion of amino acids for cancer prevention and treatment}

John DiGiovanni

Division of Pharmacology \& Toxicology, College of Pharmacy and Live STRONG Cancer Institutes, Dell Medical School, the University of Texas at Austin, Austin, TX 78712, USA.

Significant differences exist between the metabolism of normal and malignant cells. We have recently evaluated two potential therapeutic enzymes that degrade critical amino acids required for tumor growth. These engineered human enzymes, one that degrades cystine/cysteine [Cyst(e)inase] and one that degrades methionine (Methioninase), are currently under investigation for anticancer activity with promising preclinical results for prostate cancer as well as several other cancers. Depletion of extracellular cystine/cysteine leads to depletion of intracellular cysteine, decreased levels of intracellular glutathione (GSH) and increases in intracellular ROS leading to activation of cellular signaling pathways and cancer cell death. Depletion of extracellular methionine leads to reductions in intracellular $L$-methionine, $S$-adenosylmethionine and polyamines as well as reduced levels of cysteine and GSH and cancer cell death. Both enzymes given i.p. significantly reduced serum levels of their respective amino acid targets and significantly inhibited tumor growth in vivo. These and other studies on the mechanisms associated with their potential anticancer activity will be presented. In addition, studies are underway to identify agents that would synergize with one or both of these enzymes for enhanced therapeutic efficacy. Promising results have been obtained through targeting parallel antioxidant defense pathways and mitochondrial metabolism and these results will also be presented. Research supported by NIH NCI grant CA1 89623.

\section{Biography}

John DiGiovanni received his BS degree in Pharmacy and his PhD degree in Pharmacology from the University of Washington, Seattle, Washington. He did his postdoctoral work at the McArdle Laboratory for Cancer Research, University of Wisconsin, Madison, WI in carcinogenesis and cancer biology. Dr. DiGiovani joined the University of Texas at Austin (UT Austin) in January of 2010 and is Professor in the Division of Pharmacology and Toxicology, College of Pharmacy. He holds the Coulter R. Sublett Endowed Chair in Pharmacy. He also has adjunct appointments in the Department of Nutritional Sciences (College of Natural Sciences) and the Department of Pediatrics (Dell Medical School). In addition, Dr. DiGiovanni is Director of the Center for Molecular Carcinogenesis and Toxicology (CMCT) at UT Austin and is Associate Director for Basic Research in the LiveSTRONG Cancer Institutes, Dell Medical School at UT Austin. He is the Editor-in-Chief for the journal Molecular Carcinogenesis. Dr. DiGiovanni has published more than 250 research articles in prestigious peer-reviewed journals and more than 50 invited reviews/ book chapters.

\section{Cancer stem cells and miRNA in osteosarcoma}

Gaia Palmini ${ }^{1}$, Cecilia Romagnoli ${ }^{1}$, Roberto Zonefrati ${ }^{1}$, Gianna Galli ${ }^{1}$, Francesca Marini ${ }^{1}$, Alessandra Aldinucci ${ }^{2}$, Carmelo Mavilia ${ }^{1}$, Gigliola Leoncini ${ }^{1}$, Antonella Simoni ${ }^{1}$, Alessandro Franchi ${ }^{3}$, Giovanni Beltrami ${ }^{4}$, Domenico Andrea Campanacci ${ }^{5}$, Rodolfo Capanna ${ }^{3}$, Maria Luisa Brandi ${ }^{1}$

${ }^{1}$ Dipartimento di Chirurgia e Medicina Traslazionale, Università degli Studi di Firenze, Firenze 50121, Italy. ${ }^{2}$ Dipartimento di Neuroscienze, Psicologia, Area del Farmaco e Salute del Bambino, Università degli Studi di Firenze, Firenze 50121, Italy. 


\author{
${ }^{3}$ Dipartimento di Ricerca Traslazionale e delle Nuove Tecnologie in Medicina e Chirurgia, Università di Pisa, \\ Pisa 56126, Italy. \\ ${ }^{4}$ Ortopedia Oncologica Pediatrica, AOU Careggi-AOU Meyer, Firenze 50139, Italy. \\ ${ }^{5}$ SOD Ortopedia Oncologica e Ricostruttiva, AOU Careggi, Firenze 50134, Italy.
}

In the last years, several studies have identified the presence inside many types of tumors of a subpopulation of stem-like cells, a biologically unique subset of cells, which are probably involved in tumor progression and in the resistance to the antineoplastic treatments. These cells are defined as cancer stem cells (CSCs) and their uncomplete eradication represents a possible explanation as why many therapies seem to have an initial effect, but the patient later relapses. On the other hand, there are a lot of researches about the role of microRNAs (miRNAs) in several cancerous process (i.e., growth, invasion and metastases) and in the maintenance of stemness. On the base of this, we have studied the presence of CSCs inside the primary and aggressive bone malignancy which primarily affecting children and young adults, the osteosarcoma (OS). In this study, we have shown not only the presence of the CSCs population inside different high grade types of OS (i.e., telangiencatic OS, small cell OS and conventional OS), but also the preliminary results a about the possibility to find a profile of miRNA expression, as "miRNA codes", which can be analogue to all the analyzed subtypes of OS. These results could be important to identify a common molecular code among the three highly malignant forms of OS that could be used in the design of new and more effective treatments against the highly aggressive types of OS.

\title{
Biography
}

2011 she has obtained her Master's Degree in Biology at the University of Florence, Florence, Italy. 2016 she has completed her PhD from University of Florence, Florence, Italy, in the field on pathophysiology of bone. 2016-present she is a Post-Doc researcher, at the Department of Surgery and Translational Medicine, University of Florence, Florence, Italy. 2013-present she's carrying on researches on primary bone cancers (i.e., osteosarcoma, Ewing's sarcoma and giant cell tumor). Her interest are focused on cancer stem cells and on miRNAs involved in these bone diseases. She has published several papers in reputed journals and she has attended national and international meetings on this topic.

\section{Vitamin $\mathrm{E} \delta$-tocotrienol induces apoptosis in prostate cancer cells through activation of the ER stress-autophagy axis and rewiring of mitochondrial metabolism}

\section{Fabrizio Fontana, Marina Montagnani Marelli, Michela Raimondi, Roberta Manuela Moretti, Monica Marzagalli, Raffaella Longo, Maurizio Crestani, Patrizia Limonta}

Department of Pharmacological and Biomolecular Sciences, Università degli Studi di Milano, Milano 20133, Italy.

Prostate cancer is androgen dependent in its early stage and responds to the androgen deprivation therapy; however, it often progresses to the castration-resistant prostate cancer (CRPC) stage in which cancer cells become resistant to therapies. Preliminary experiments performed in the authors' laboratory demonstrate that vitamin E-derivative $\delta$-tocotrienol $(\delta$-TT) exerts a proapoptotic activity in CRPC cells while sparing normal prostate epithelial cells. These studies were performed to investigate the molecular mechanisms underlying this activity: (1) in CRPC cells, $\delta$-TT induces endoplasmic reticulum (ER) stress (expression of ER stress markers such as BiP, PERK, p-eIF2 $\alpha$, IRE1 $\alpha$, ATF4 and CHOP) and autophagy (expression of autophagy markers such as LC3- II and p62). Using the ER stress inhibitors salubrinal and 4-PBA and the autophagy flux inhibitors 3-methyladenine and chloroquine, we confirmed that the effect of $\delta$-TT is 
mediated by both these mechanisms. Moreover, treatment of CRPC cells with salubrinal impairs $\delta$-TTinduced LC3-II expression, demonstrating that this compound triggers the ER stress-autophagy axis; (2) in CRPC cells, the expression levels of the proteins of the respiratory chain complexes are increased compared to non-tumoral cells, supporting a reprogramming of the OXPHOS system. In these cells, $\delta$-TT: decreases the expression levels of the proteins of the respiratory chain complexes, specifically of complex I; decreases the mitochondrial activity as well as the mitochondrial activity/mass ratio; decreases both ATP production and oxygen consumption. These data demonstrate that $\delta$-TT exerts a significant proapoptotic activity in CRPC cells through activation of the ER stress-autophagy axis and rewiring of mitochondrial metabolism.

\section{Biography}

Patrizia Limonta has completed her post-graduate degrees in Pharmacology and in Experimental Endocrinology. She is full professor of Applied Biology at the Department of Pharmacological and Biomolecular Sciences, Università degli Studi di Milano, Milano, Italy. She has published more than 90 papers in reputed journals and has been serving as an editorial board member of Frontiers in Endocrinology (Cancer Endocrinology Section), Recent Patents in Anticancer Drug Discovery, Oncology Letters, Clinical Cancer Drugs.

\section{Targeting metabolic dysfunction in cancer cells by combinatorial treatment with natural compounds}

Alessia Lodi', Achinto Saha ${ }^{2}$, Xi-Yuan Lu1, Bo Wang ${ }^{1}$, Enrique Sentandreu', Meghan Collins ${ }^{1}$, Mikhail G. Kolonin $^{3}$, John DiGiovanni ${ }^{2,4}$, Stefano Tiziani ${ }^{1,4}$

${ }^{1}$ Department of Nutritional Sciences, University of Texas at Austin, Austin, TX 78712, USA.

${ }^{2}$ Division of Pharmacology and Toxicology, College of Pharmacy, University of Texas at Austin, Austin, TX 78712, USA.

${ }^{3}$ The Brown Foundation Institute of Molecular Medicine, University of Texas Health Science Center at Houston, Houston, TX 77030, USA.

${ }^{4}$ Dell Pediatric Research Institute, University of Texas at Austin, Austin, TX 78712, USA.

Natural compounds ingested through the diet can modulate key molecular signaling, inhibit cancer cell proliferation and induce apoptosis. According to the World Health Organization, an increased consumption of natural compounds could prevent about one-third of all cancer deaths. Libraries of natural compounds incorporate phytochemicals characterized by vast molecular diversity and are essential to identify combinations that synergistically contribute to improving treatment outcome. Given the very large number of possible combinations, high-throughput screening methods are paramount to identify the most efficacious compound combinations. In this study, we used high-throughput screening to test the outcome of natural compound combinations on prostate cancer. The in vitro screen identified ursolic acid, curcumin and resveratrol as top-hits. These compounds were then administered via the diet to a mouse allograft model of prostate cancer. Combinations of the compounds greatly improved outcome, in vivo. This outcome was, at least in part due to the modulation of glutamine metabolism, as determined via untargeted metabolomics and metabolic flux analysis, induced by compound combinations. Moreover, the combinations affected levels of ASCT2 and activation of STAT3, mTORC1 and AMPK. Overall, the high throughput approach is effective for identifying combinations of natural compounds that can synergistically contribute to chemoprevention and therapeutic outcome. 
30. The effects of water extract of Ruta graveolens on glioblastoma and melanoma cells is selective and depends on REST/NRSF expression

Luca Colucci D’Amato', Maria Teresa Gentile', Olga Pastorino', Adriana Bajetto ${ }^{2}$, Asa Fex-Svenningsen ${ }^{3}$, Tullio Florio ${ }^{2}$

${ }^{1}$ Laboratory of Molecular and Cellular NeuroPathology, Di.S.T.A.Bi.F., University of Campania "L. Vanvitelli", Caserta 81100, Italy.

${ }^{2}$ Sezione di Farmacologia, Dipartimento di Medicina Interna \& Centro di Eccellenza per la Ricerca Biomedica (CEBR), Università di Genova, Genova 16132, Italy.

${ }^{3}$ Department of Neurobiology Research, University of Southern Denmark, Odense DK-5000, Denmark.

Heterogeneity and recurrent relapse of tumors, drugs resistance and the lack of selectivity of current chemotherapy calls for new drugs.

Here we report that RGWE is able to kill a number of glioblastoma (GBM) cell lines and cancer stem cells as shown by MTT and trypan blue assay. In particularly, we analyzed U87MG, U138, C6 cell lines as well as 3 cancer stem cells originated from patients affected by GBM. RGWE, differently to temozolomide and cisplatin, does not show any toxic activity towards differentiated neurons. Interestingly, differently to GBM cells responsive to RGWE, T98G GBM cell line, unresponsive to RGWE, do not express the transcription factor REST/NRSF, encoding a zinc finger protein that function as a master regulator of neural cell differentiation in normal physiological conditions. NRSF is highly expressed in embryonic stem cells but reduced rapidly in neuron progenitors and maintained at very low levels after differentiation. In contrast, in neuroepithelial tumors, high levels of NRSF are expressed in medulloblastomas, neuroblastomas and multiform glioblastoma and are correlated with the proliferation and severity of these tumors. We observed that down regulation of REST/NRSF in U87MG and C6 cells by means of siRNA, prevents RGWE cytotoxic effects.

We also found that human melanoma cell lines, HNCB and A375 expressing high and low levels of REST/ NRSF respectively, respond differently to RGWE. HNCB (REST ${ }^{\text {high }}$ ) cells die upon RGWE administration whereas A375 (REST ${ }^{\text {low }}$ ) cells are unresponsive. RGWE stimulation is able to affect also cell migration as shown by wound assay. Upon siRNA-mediated REST down-regulation, HNCB cells show unresponsiveness to RGWE's effects.

Finally, we evaluated the effects of RGWE in the tube formation assay on Matrigel, an angiogenic assay. We found that RGWE is able to impair tubule network formation when administered to human endothelial cells, HUVEC.

\section{A natural agent bitter melon exerts strong efficacy against pancreatic cancer in combination} with gemcitabine in patient derived xenografts

\section{Rajesh Agarwal}

Department of Pharmaceutical Sciences, University of Colorado Skaggs School of Pharmacy and Pharmaceutical Sciences, Colorado 80045, USA.

Pancreatic cancer (PanC) remains the 4th leading cause of cancer related-deaths in U.S. resulting in a dismal survival rate of $<5 \%$. This generates a critical need for identifying novel non-toxic agents aimed at 
effective PanC management with minimal patient distress. Bitter melon (Momordica charantia), a dietary agent, is actively being examined for its anti-cancer efficacy against a panel of malignancies. Recent data from our group highlights the anti-PanC potential of bitter melon juice (BMJ); its role in PanC-cancer stem cell (CSC) self-renewal/ kinetics and gemcitabine (GEM) resistance in PanC cells. Combinations for $\mathrm{BMJ}$ and GEM in vitro also showed synergism in AsPC1 and synergistic effect in BxPC3 PanC cells. As patient-derived xenografts are gaining importance, we next assessed the efficacy of BMJ and GEM alone, or in combination; tumor growth was significantly inhibited in all treated groups with GEM and Combo displaying comparable efficacies followed by BMJ. Notably, tumor regrowth determination with subsequent treatment washout period showed maximum tumor regrowth in GEM group comparable to controls; however, BMJ consistently showed a prolonged inhibitory effect and Combo group remained close to BMJ, suggesting no apparent antagonistic interference. In mechanistic evaluations, in tumor tissues, minimal expression levels of Ki67 and VEGF were observed in BMJ and Combo groups by study end compared to controls and GEM groups. Together, these results show strong BMJ efficacy as a single agent as well as being a perfect combinatorial candidate with GEM for PanC management and treatment. Supported by NCI R01 grant CA195708.

\title{
Biography
}

Rajesh Agarwal completed his Ph.D. from Lucknow University, India in 1981. He is currently Professor and Vice Chairman, Department of Pharmaceutical Sciences, University of Colorado. He has over 360 peer-reviewed publications and several book chapters, has been an invited speaker across the globe, has over three hundred presentations in national and international scientific meetings, is an active member of several National Institutes of Health, USA grant review committee, and is an editorial board member of several lead cancer journals.

\section{The mitochondrial-targeted compound SS-31 improves cachexia by increasing muscle energetics}

\author{
Riccardo Ballarò ${ }^{1,2}$, Marc Beltrà ${ }^{1,2}$, Paola Costelli, ${ }^{1,2}$, Hazel Szeto $^{3}$, Fabio Penna ${ }^{1,2}$ \\ ${ }^{1}$ Department of Clinical and Biological Sciences, Experimental Medicine and Clinical Pathology Unit, \\ University of Turin, Turin 10126, Italy. \\ ${ }^{2}$ Interuniversity Institute of Myology, Milano 20132, Italy. \\ ${ }^{3}$ Weill Cornell Medicine, New York, NY 10016, USA.
}

Cachexia is a complex syndrome that frequently complicates the management of cancer patients ${ }^{[1]}$. This syndrome is characterized by progressive wasting of body mass, mainly due to the depletion of both adipose and muscle tissue ${ }^{[2]}$. Together with increased protein degradation, also mitochondrial impairment likely contributes to muscle wasting ${ }^{[3]}$. Indeed, alterations of mitochondrial morphology and function have been observed in the skeletal muscle of tumor-bearing animals with cachexia ${ }^{[3]}$.

The aim of the present study was to investigate if selective targeting of mitochondria with SS-31 could exert positive effects on muscle wasting in mice bearing the C26 tumor treated or not with chemotherapy (oxaliplatin+5-fluorouracil; C26 and C26 OXFU, respectively).

Both C26 hosts and C26 OXFU mice showed anorexia, body weight loss and decreased muscle mass and strength. In the C26 hosts, SS-31 partially prevented body wasting, anorexia and muscle mass. In the 
skeletal muscle of both $\mathrm{C} 26$ and $\mathrm{C} 26$ OXFU animals, mitochondrial markers such as PGC-1 $\alpha$, cytochrome $\mathrm{c}$ and SDHA were reduced and these alterations were not corrected by SS-31. In the C26 OXFU mice the reduced expression of mitochondrial markers was also associated with impaired muscle oxidative capacity and ATP content. These latter were improved by SS-31 in the C26 hosts but not in the C26 OXFU mice. Muscle wasting and mitochondrial impairments were associated with reduced protein synthesis in the C26 hosts. Consistently with improved muscle energetics, SS-31 administration partially corrected muscle protein synthesis in C26-bearing mice.

Overall, SS-31 administration could contribute to correct muscle wasting and to improve muscle energetics in tumor-bearing animals, suggesting that targeting mitochondria could be part of a multimodal therapy against cancer cachexia. In order to increase the translational value of the results, further analysis and treatment optimization are needed in order to demonstrate the effectiveness of SS-31 in C26-bearing mice treated with chemotherapy.

\title{
REFERENCES
}

1. von Haehling S, Anker MS, Anker SD. Prevalence and clinical impact of cachexia in chronic illness in Europe, USA, and Japan: facts and numbers update 2016. J Cachexia Sarcopenia Muscle 2016;7:507-9.

2. Argilés JM, Busquets S, Stemmler B, López-Soriano FJ. Cancer cachexia: understanding the molecular basis. Nat Rev Cancer. Nat Rev Cancer 2014; 14:754-62.

3. Pin F, Busquets S, Toledo M, Camperi A, Lopez-Soriano FJ, et al. Combination of exercise training and erythropoietin prevents cancerinduced muscle alterations. Oncotarget 2015;6:43202-15.

\section{Resveratrol counteracts the pro-invasive activity of lysophosphatidic acid in ovarian cancer cells by rescuing autophagy and down-regulating the hedgehog pathway}

\author{
Alessandra Ferraresi', Christian Seca', Suyanee Thongchot ${ }^{1}$, Letizia Vallino', Giovanna Chiorino ${ }^{2}$, Danny N. \\ Dhanasekaran ${ }^{3}$, Ciro Isidoro ${ }^{1}$
}

${ }^{1}$ Laboratory of Molecular Pathology and Nanobioimaging, Department of Health Sciences, Università del Piemonte Orientale "A. Avogadro", Novara 28100, Italy.

${ }^{2}$ Cancer Genomics Laboratory, Fondazione Edo ed Elvo Tempia, Biella 13900, Italy.

${ }^{3}$ Stephenson Cancer Center and Department of Cell Biology, The University of Oklahoma Health Sciences Center, Oklahoma City, OK 73104, USA.

Ovarian cancer emerges as a highly aggressive metastatic disease characterized by a high grade of lethality and it remains the most lethal gynecologic cancer with a five-year survival rate of about $30 \%-40 \%$. Cancer progression is facilitated by pro-invasive factors released by the tumour microenvironment and modulating the epithelial-to-mesenchymal transition (EMT).

Lysophosphatidic acid (LPA), a bioactive phospholipid highly secreted in the ascitic fluid and plasma of ovarian cancer patients, stimulates the growth and promotes the tissue invasion of cancer cells. Resveratrol (RV), a polyphenol found in grapes and in a variety of natural products, is attracting the interest of many researchers thank to its several anti-cancer properties.

Here we show that LPA induces the EMT through induction of the Hedgehog pathway and concomitant inhibition of autophagy in the cancer cells at the migration front. We found that RV and LPA regulate in an 
opposite fashion the expression of BMI-1, a Polycomb transcriptional repressor belonging to the Hedgehog pathway, involved in cancer cell stemness and metastasis. Interestingly, BMI-1 silencing restores autophagy and halts ovarian cancer cell migration. Our data indicate that there is a functional cross-talk between autophagy and Hedgehog/EMT/cell migration processes and we propose BMI-1 as the joining mediator.

In conclusion, our findings indicate that RV elicits its anti-tumor effect through induction of autophagy and down-regulation of BMI-1, and that this process is sufficient to inhibit cancer cell migration and invasion.

\title{
34. Effects of HDAC inhibitors on glioblastoma cells
}

\author{
Olga Pastorino ${ }^{1,2}$, Maria Teresa Gentile ${ }^{1}$, Adriana Bajetto ${ }^{3}$, Antonella Di Costanzo ${ }^{4}$, Alessandro Mancini ${ }^{5,6}$, \\ Lucia Altucci ${ }^{4}$, Tullio Florio ${ }^{3}$, Maria Patrizia Stoppelli ${ }^{2}$, Luca Colucci D’Amato ${ }^{1}$ \\ ${ }^{1}$ Laboratory of Molecular and Cellular NeuroPathology, Di.S.T.A.Bi.F., University of Campania "L. \\ Vanvitelli", Caserta 81100, Italy. \\ 2Istituto di Genetica e Biofisica "ABT", CNR, Naples 80131, Italy. \\ ${ }^{3}$ Sezione di Farmacologia, Dipartimento di Medicina Interna \& Centro di Eccellenza per la Ricerca \\ Biomedica (CEBR), Università di Genova, Genova 16132, Italy. \\ ${ }^{4}$ Dipartimento di Medicina di Precisione, University of Campania "L. Vanvitelli", Caserta 81100, Italy. \\ ${ }^{5}$ Dipartimento di Scienze Mediche Traslazionali, University of Campania "L. Vanvitelli", Caserta 81100, Italy. \\ ${ }^{6}$ BIOUP Sagl, Lugano 6900, Switzerland.
}

Background: Glioblastoma Multiforme (GBM), a high-grade glioma (WHO grade IV), is the most aggressive form of brain cancer. Treatment options for GBM involving a combination of surgery, chemotherapy and radiation resulted in a poor survival outcome. Epigenetic mechanisms are increasingly implicated in GBM pathogenesis. Unlike genetic mutations, epigenetic changes arereversible and can be targeted by drugs. We evaluated whether different histone deacetylase Inhibitors (HDACis) are able to affect migration, invasion and vasculogenic mimicry in GBM cells.

Methods: We tested Varinostat (SAHA) and Trichostatin A (TSA) as inhibitors of class I and II HDACs, Entinostat (MS275) as selective inhibitor of class I HDACs (specifically of HDAC 1 and 3) and MC1568 as selective HDAC class II inhibitor. Tube Assay was used to evaluate the ability of HDAC is to interfere with vessels formation by human GBM U87MG and rat glioma C6 cell lines and by cancer stem cells (CSCs) isolated from human GBMs. U87MG directional cell migration and cell invasion have been assessed by Boyden chamber assay and using xCELLigence Real-Time Cell Analyzer. Trypan Blue exclusion test and MTT assay were employed to assess cell viability and cell proliferation.

Results: Our data show that sublethal doses of HDACis significantly decrease U87MG directional cell migration and that MS275 HDACi is able to impair U87MG matrigel invasion. Finally, we observed that HDACis inhibit significantly vasculogenic mimicry in U87MG, C6 and CSCs cells.

Conclusion: GBM depends on vascular networks to supply blood, oxygen, and nutrients. Tumor blood vessels can either be formed from pre-existing blood vessels (neo-angiogenesis) or from tumor cells (vasculogenic mimicry) due to a process of epithelial-mesenchymal transition; vascular mimicry provides a mechanism whereby GBM could escape anti-angiogenic therapies. Our results suggest that HDACis may be promising candidates for blocking vascular mimicry. 


\title{
35. Resveratrol and Halofuginone Counteract TGF- $\beta$-Induced activation and proliferation of stromal fibroblasts by Up-regulating Autophagy
}

\author{
Eleonora Secomandi', Sami Ibazizen², Gwenael Rolin², Ciro Isidoro \\ ${ }^{1}$ Laboratory of Molecular Pathology and Nanobioimaging, Department of Health Sciences, Università del \\ Piemonte Orientale, Novara 28100, Italy. \\ ${ }^{2}$ Inserm UMR 1098, Engineering and Cutaneous Biology Team, BesanÇon 25020, France.
}

Cancer can be viewed as a wound that never heals. In this respect, keloids can be considered as the "benign" counterparts of cutaneous tumors. Keloids are pathological scars derived from an altered skin wound healing process and are characterized by hyper-activation and abnormal proliferation of stromal fibroblasts. Among the several pro-inflammatory cytokines involved in the pathogenesis of keloids, transforming growth factor- $\beta$ (TGF- $\beta$ ) plays a crucial role in myofibroblast proliferation and differentiation. Resveratrol (RV), a naturally polyphenolic compound, is a strong autophagy inducer and inhibits oxidation, inflammation and collagen I deposition. Halofuginone (HF) is a synthetic halogenated derivate of febrifugine, well known for its anti-fibrotic therapeutic potential.

In the present work, we focused our attention on the role of autophagy in keloid formation and progression. We found that RV and HF counteract TGF- $\beta$-induced migration of keloid fibroblasts and, in parallel, an increase of autophagic flux is detected, suggesting autophagy as a putative mechanism responsible for slowing down cell motility. Moreover, HF reduces the number of Ki67+ cells, decreasing the proliferative capacity of keloid fibroblasts. Both RV and HF attenuate $\alpha$-SMA and COL1 synthesis and limit the contraction capability of myofibroblasts within the collagen matrix, even in co-treatment with TGF- $\beta$.

\section{The possible mechanism of the antioxidants on the antitumor effect of bortezomib in melanoma and myeloma cell lines}

\author{
Angéla Takács ${ }^{1}$, Eszter Lajkó1, Ildikó Istenes² ${ }^{2}$ László Kőhidai ${ }^{1}$, Orsolya Láng ${ }^{1}$ \\ ${ }^{1}$ Department of Genetics Cell- and Immunobiology, Semmelweis University, Budapest 1085, Hungary. \\ ${ }^{2}$ Department of Internal Medicine, Semmelweis University, Budapest 1085, Hungary.
}

Bortezomib is a proteasome inhibitor chemotherapeutic agent used to treat multiple myeloma; recently studies offer it as a promising drug to treat melanoma. Bortezomib-induced peripheral neuropathy (BIPN) is a dose-limiting side-effect, that can be treated with antioxidants, e.g., alpha-lipoic acid and thiamine.

The objective of our experiments was: to verify the cytotoxicity of bortezomib; to test and compare the influence of the antioxidants on the antitumor effect of bortezomib in melanoma and myeloma.

The cells were U266 myeloma, WM35 primary and A2058 metastatic melanoma. The concentrations were: (1) bortezomib: 20, 100 and $300 \mathrm{ng} / \mathrm{mL}$; (2) alpha-lipoic acid: 10 and $100 \mu \mathrm{g} / \mathrm{mL}$ (3) thiamine: 150 and $300 \mathrm{nmol} / \mathrm{L}$. Impedimetry (xCELLigence SP) was used to evaluate the cytotoxicity. To study the cell cycle profile, Cell-ClockTM, a redox dye was applied and evaluated by ImageJ. Apoptosis was analyzed by flow cytometry after annexin $\mathrm{V}$ assay and phospho-p53 immunostaining.

Bortezomib was cytotoxic and blocked U266 cells in the G1 phase. Alpha-lipoic acid enhanced the cytotoxicity of bortezomib $(20 \mathrm{ng} / \mathrm{mL})$ in U266 cells, but counteracted bortezomib in melanoma cells. 
Thiamine (300 nmol/L) with bortezomib antagonized the antitumor effect of bortezomib on myeloma cells. In every cell, bortezomib was apoptotic, which effect was decreased in melanoma by the co-treatments.

A good correlation between the apoptotic and the cell cycle arresting effect of bortezomib as well as the antagonism of the antioxidants was found. In conclusion, the combination therapy of bortezomib and a low dose of antioxidants could be offered for the treatment of BIPN in multiple myeloma.

\section{Biography}

Angéla Takács received the Doctor of Pharmacy degree from the Semmelweis University in 2017. Currently, she is a PhD student in the Modern Trends in Pharmaceutical Scientific Research Program at the Department of Genetics Cell- and Immunobiology, Semmelweis University. She is woking on projects in connection with targeted tumor therapy.

\section{Profiling of the transcripts and microRNA in ovarian cancer cells subjected to fasting or to the caloric restriction mimetic Resveratrol}

Letizia Vallino ${ }^{1}$, Alessandra Ferraresi ${ }^{1}$, Chiara Vidoni ${ }^{1}$, Giovanna Chiorino ${ }^{2}$, Alessandra Galetto ${ }^{3}$, Ciro Isidoro'

${ }^{1}$ Laboratory of Molecular Pathology, Department of Health Sciences, Università del Piemonte Orientale "A. Avogadro", Novara 28100, Italy.

${ }^{2}$ Cancer Genomics Laboratory, Fondazione Edo ed Elvo Tempia, Biella 13900, Italy.

${ }^{3}$ Oncology Unit, Department of Translational Medicine, Università del Piemonte Orientale "A. Avogadro", Novara 28100, Italy.

The lack of nutrients and of growth factors has a great impact on the metabolic pathways involved in cancer cell proliferation. In this condition, cancer cells activate autophagy as a pro-survival pathway and eventually exit the cell cycle to undergo a dormant state. Presently, the potential benefit of nutrient starvation in the prevention and treatment of cancer is under clinical consideration. Resveratrol (RV), a dietary polyphenol acting as a protein (caloric) restriction mimetic, could substitute for amino acid starvation. Here we report on the changes of the global transcriptome in ovarian cancer cells subjected to amino acid starvation or to the protein-caloric restriction mimetic RV. Based on the Gene Ontology analysis, the mRNAs expressed in the two treatments affected different biological functions. The number of transcripts positively impinging on the autophagy pathway was higher in RV-treated than in starved cancer cells. Our data support the view that RV treatment can be more effective than and can substitute for nutrient starvation to induce a dormant-like state in cancer cells.

\section{Resveratrol abrogates IL-6 - induced ovarian cancer cell motility by limiting glucose availability and up-regulating autophagy}

Chiara Vidoni ${ }^{1}$, Alessandra Ferraresi $^{1}$, Suratchanee Phadngam ${ }^{1}$, Andrea Castiglioni ${ }^{1}$, Alessandra Galetto ${ }^{2}$, Ciro Isidoro ${ }^{1}$

${ }^{1}$ Laboratory of Molecular Pathology and Nanobioimaging, Department of Health Sciences, Università del Piemonte Orientale "A. Avogadro", Novara 28100, Italy. 
${ }^{2}$ Unit of Oncology, Department of Translational Medicine, Università del Piemonte Orientale "A. Avogadro", Novara 28100, Italy.

In this work, we investigated whether glucose uptake plays a role in ovarian cancer cell migration. IL-6, a cytokine released by cancer-associated fibroblasts and cancer cells, promotes ovarian cancer metastasis. We have previously found that Resveratrol (RV), a naturally polyphenolic compound, counteracts IL-6induced cancer cell migration, through the epigenetic up-regulation of autophagy. Here, we show that IL- 6 stimulates glucose-uptake along with cell migration. We found that in the presence of glucose, IL-6-induced mitochondrial oxidative stress with production of anion superoxide and inactivation of ATG4, the cysteine protease that processes LC3 needed for autophagosome formation. RV reduced glucose uptake, prevented the formation of anion superoxide, rescued ATG4 function and increased the level of autophagosome formation. RV-mediated up-regulation of autophagy was more pronounced at the migration front, leading to a reduced cancer cell motility. These data indicate that IL- 6 promotes ovarian cancer cell migration by promoting glucose uptake which is metabolized to produce mitochondrial oxidative stress that eventually inhibits autophagy, and RV may counteract this action by limiting the glucose uptake and the production of anion superoxide.

\section{Warty Carcinoma of the uterine cervix - do we know it}

\section{Angel Yordanov ${ }^{1}$, Strahil Strashilov ${ }^{2}$, Milena Karcheva ${ }^{3}$, Polina Vasileva ${ }^{4}$, Ilko lliev ${ }^{1}$, Stanislav Slavchev ${ }^{5}$, Yoana Ivanova ${ }^{4}$}

${ }^{1}$ Clinic of Gynecologic Oncology, Medical University Pleven, Pleven 5803, Bulgaria.

${ }^{2}$ Department of Plastic Restorative, Reconstructive and Aesthetic Surgery, Medical University Pleven, Pleven 5803, Bulgaria.

${ }^{3}$ Department of Epidemiology, Medical University Pleven, Pleven 5803, Bulgaria.

${ }^{4}$ Department of Obstetrics and Gynecology, Medical University Pleven, Pleven 5803, Bulgaria.

${ }^{5}$ Clinic of Gynaecology, University Hospital "St. Anna"-Varna, Varna 9002, Bulgaria.

Introduction: Warty carcinoma of the uterine cervix is a rare subtype of squamous cell carcinoma that usually occurs in women over 48-50 years. Warty carcinoma is not as aggressive as the other subtypes, and has a better prognosis. It is also a slow-growing tumor. The prognosis depends on a set of several factors that include: the size of the tumor and the extent of its invasion, stage of cancer, FIGO grade, involvement of the regional lymph nodes, whether the tumor is occurring for the first time, or is a recurrent tumor, response to treatment.

Aim: The aim of the study was to explore the prognosis of this type of cervical cancer.

Methods: For ten year period (2008-2017) in the Clinic of Gynecologic oncology at the UMHAT - Pleven, Bulgaria were operated 714 cases with Carcinoma of the uterine cervix and 14 of which were histologically diagnosed as a Warty carcinoma. Radical abdominal hysterectomy was performed on all patients with pelvic lymph node dissection with postoperative telegamma therapy. Patients were investigated by retroand prospective analysis for overall and recurrence-free survival rate.

Results: Warty carcinoma was $1.94 \%$ of all cervical carcinomas, treated in our clinic. The mean age of the patients was 48 years ( 29 to 72 years) and $57 \%$ of them were peri- and postmenopausal and $43 \%$ were in women under the age of 45 . According to the FIGO staging systems for cervical cancer, patients were staged as follows: in stage IB1 - 43\% and in stage IB2 - 57\%. In $29 \%$ the tumor size was under $2 \mathrm{~cm}$, in $14 \%$ 
the size was $2-4 \mathrm{~cm}$ and in $57 \%$ the size of the local tumor is above $4 \mathrm{~cm}$. Never the less the high percentage of locally advanced process (patients with tumor above $4 \mathrm{~cm}$ ), only in one case there was local spreading of the tumor to the uterine cavity and in one case there lymph node metastasis. Lymphovascular space invasion was not seen in any patient. Our patients are free of tumor recurrence or occurrence of symptoms for a period of 9.4 years (112 months), to 4 months after the surgical procedure.

Conclusion: Warty carcinoma of the cervix has good clinical prognosis and it is possible to rethink for not so aggressive surgical treatment and even for fertility sparing surgery when it is necessary.

\title{
40. Peptide based targeted drug delivery of mono- and dual-drug conjugates to prostate cancer cells
}

\author{
Michael Firer ${ }^{1,2}$, Oranit Bashari', Boris Redko ${ }^{3}$, Anna Cohen ${ }^{1}$, Galia Luboshits ${ }^{1}$, Gary Gellerman ${ }^{2,3}$ \\ ${ }^{1}$ Department of Chemical Engineering, Ariel University, Ariel 40700, Israel. \\ ${ }^{2}$ Ariel Center for Applied Cancer Research, Ariel University, Ariel 40700, Israel. \\ ${ }^{3}$ Department of Chemical Sciences, Ariel University, Ariel 40700, Israel.
}

Targeted drug delivery (TDD) systems are becoming important in cancer therapy for the specific delivery of cytotoxic drugs into the target cells. This strategy bypasses the disadvantages of traditional chemotherapy such as non-specific toxicity and development of drug resistance and may also reduce drug doses. TDD systems commonly consist of a targeting moiety (such as an antibody, protein or peptide) chemically conjugated to a linker which has been coupled to a cytotoxic compound. In order for targeted nanoparticles to be effective therapeutics, a number of design issues need to be considered. This presentation will discuss selection of appropriate peptides derived from phage display libraries as targeting moieties, focusing on our results in the development of peptide-drug-conjugates (PDCs) for prostate cancer therapy. Based on our previous studies in TDD systems, we defined a series of phage selection criteria which enabled the choice of candidate peptides for testing in vitro and in vivo. Using the selection criteria, two lead peptides, P10 and P12 were used to build mono- and dual-drug PDCs which were shown to be target specific and effective against a variety of prostate cancer cells. These results demonstrate several important principles in the tailored design and selection of both the peptides and cytotoxic drugs suitable for incorporation into effective PDCs. We are now using these principles to design PDCs for other cancers.

\section{Pregnancy-associated breast cancer is it always possible to keep the pregnancy}

\section{Polina Vasileva ${ }^{1}$, Angel Yordanov ${ }^{2}$, Vasil Nanev ${ }^{3}$, Strahil Strashilov ${ }^{4}$, Ilko Iliev ${ }^{2}$, Elitsa Gyokova ${ }^{1}$, Yoana Ivanova-Yoncheva ${ }^{1}$}

\footnotetext{
${ }^{1}$ Department of Obstetrics and Gynecology, Medical University-Pleven, Pleven 5803, Bulgaria.

${ }^{2}$ Department of Gynecologic Oncology, Medical University-Pleven, Pleven 5803, Bulgaria.

${ }^{3}$ Department of Surgical Oncology, Medical University-Pleven, Pleven 5803, Bulgaria.

${ }^{4}$ Department of Surgical Diseases, Medical University-Pleven, Pleven 5803, Bulgaria.
}

Introduction: Pregnancy-associated breast cancer can occur in any moment during gestation, lactation, or even within 1 year after delivery. The incidence of that cancer is 1:3000 to 1:10000 pregnancies. The diagnosing process is often hard due to the pregnancy-related physiological changes of the breast. 
Case presentation: We present the case of a 41 -year old woman, diagnosed with breast cancer during pregnancy, in 20th week of gestation; the cancer was found due to presence of multiple pathological fractures of the spinal cord.

Discussion: Despite the fact that treating breast cancer during pregnancy is done following the same principals as the management of breast cancer in non-pregnant patientstakingintoaccountthat the aim of the treatment is to provide the best curative treatment with minimal or no harm to the fetus maximizing the gestational period and ensure safe delivery of the fetus, is not always easy to keep the pregnancy.

Conclusion: In conclusion, with the increasing birth age for women, PABC will be a more common disease. We have to focus our thinking on it. Elective termination of pregnancy has not proven to improve the outcome in breast cancer. Therefore, it is not routinely recommended. The decision to terminate has to be individualized based on the oncologic situation and maternal concerns.

\title{
Biography
}

Vasileva PP has completed her graduation from Medical University-Pleven, Bulgaria in 2011. Since 2012 he has been working in Clinic of Obstetrics and gynecology, University Hospital “Dr.Georgi Stranski”-Pleven, Bulgaria. She has several publications in various magazines on different topics. She has published more than 10 papers in reputed journals.

\section{Mild exercise training partially prevents muscle wasting induced by tumor and chemotherapy}

\author{
Riccardo Ballarò ${ }^{1,2}$, Marc Beltrà ${ }^{1,2}$, Fabrizio Pin ${ }^{1,2}$, Kia Ranjbar $^{3}$, Paola Costelli ${ }^{1,2}$, Fabio Penna ${ }^{1,2}$ \\ ${ }^{1}$ Department of Clinical and Biological Sciences, Experimental Medicine and Clinical Pathology Unit, \\ University of Turin, Turin 10126, Italy. \\ ${ }^{2}$ Interuniversity Institute of Myology, Milano 20132, Italy. \\ ${ }^{3}$ Tarbiat Modares University, Tarbiat Tehran 14117, Iran.
}

Cachexia is a frequent feature in cancer patients. Its occurrence is associated with reduced tolerance to anticancer treatments, impaired survival and bad quality of life. This syndrome is mainly characterized by loss of body weight, depletion of muscle mass and metabolic alterations ${ }^{[1]}$. The loss of muscle mass and function is also associated with autophagy deregulation and mitochondrial impairment ${ }^{[1]}$ that could be exacerbated by anti-cancer treatments ${ }^{[2]}$. In this regard, exercise could represent a promising non-pharmacological approach to correct muscle wasting in cancer cachexia ${ }^{[3]}$. The main goal of the present study was to investigate if mild exercise could improve muscle wasting in mice bearing the $\mathrm{C} 26$ tumor, in the presence or in the absence of chemotherapy treatment [oxaliplatin+5-fluorouracil (OXFU)]. Although chemotherapy increased the lifespan of $\mathrm{C} 26$-bearing mice, it resulted in muscle wasting more severe than that observed in the untreated C26 hosts. Such wasting pattern was associated with increased levels of molecules accepted as markers of autophagy and mitophagy, while the expression of markers of mitochondrial content and dynamics decreased. Consistently, C26 OXFU mice showed impaired muscle oxidative metabolism (SDH total activity) and reduced protein synthesis. Moderate exercise in OXFU-treated C26-bearing mice exerted beneficial effects on the loss of both muscle mass and function, partially restoring the expression of markers of autophagy and mitophagy, increasing mitochondrial content and dynamics and improving muscle oxidative capacity. 
On the whole, chemotherapy administration could contribute to muscle wasting in cancer patients, enhancing the alterations of metabolism due to tumor growth. In this regard, moderate exercise may support cancer patients under chemotherapy regimen preserving muscle mass and function.

\section{REFERENCES}

1. Argilés JM, Busquets S, Stemmler B, López-Soriano FJ. Cancer cachexia: understanding the molecular basis. Nat Rev Cancer 2014;14:754-62.

2. Barreto R, Waning DL, Gao H, Liu Y, Zimmers TA, et al. Chemotherapy-related cachexia is associated with mitochondrial depletion and the activation of ERK1/2 and p38 MAPKs. Oncotarget 2016;7:43442-60.

3. Pin F, Busquets S, Toledo M, Camperi A, Lopez-Soriano FJ, et al. Combination of exercise training and erythropoietin prevents cancerinduced muscle alterations. Oncotarget 2015;6:43202-15.

\section{Mouse mammary carcinomas secrete extracellular vesicles - in vitro and in vivo study}

\section{Yuko Ito, Nabil Eid, Masa-Aki Shibata, Yoshinori Otsuki, Yoichi Kondo}

Department of Anatomy \& Cell Biology, Division of Life Sciences, Osaka Medical College, 2-7 Daigakumachi, Takatsuki, Osaka, 569-8686, Japan

Many types of cells including cancer cells secrete extracellular vesicles (EVs). EVs are small vesicles released by donor cells that can be taken up by recipient cells. EVs contain receptor proteins, proteolytic enzymes, miRNAs, and mRNAs which are transferred into the target cells, and then affect various cell functions. EVs are categorized depending upon where in the cells they originate: vesicles that are derived from multivesicular bodies are referred to as exosomes (50-100 nm in diameter) and those from the plasma membranes as microvesicles (500-1000 $\mathrm{nm}$ in diameter). There are numerous in vitro studies, however, little is known about EVs in vivo. Here we show the chemical and ultrastructural features of EVs derived from cultured mouse mammary carcinoma cells and their tumors in mice formed upon inoculation. BJMC338 tumors show a low metastatic propensity, while BJMC3879 tumors show a high metastatic propensity, especially to lymph nodes and lungs. Both EVs contain premature vascular endothelial cell growth factor $\mathrm{C}$, and inoculated tumors demonstrated that EVs were in their lumen and surrounding connective tissue. Many exosomes were accumulated in the cytoplasm, Golgi complex and r-ER of tumor cells. These observations suggest that mouse mammary carcinoma cells in tumors secrete EVs.

\section{Epithelial splicing regulatory protein 1 expression is an unfavorable prognostic factor in prostate cancer patients}

Kang Hyun Lee', Sung Han Kim', Andy Jinseok Lee ${ }^{2}$, Weon Seo Park ${ }^{3}$, Jongkeun Park ${ }^{2}$, Jongkeun Lee², Boram Park ${ }^{4}$, Jae Young Joung ${ }^{1}$, Dongwan Hong ${ }^{2}$

${ }^{1}$ Department of Urology, Center for Prostate Cancer, Goyang-si 410-769, South Korea.

${ }^{2}$ Clinical Genomics Analysis Branch, National Cancer Center Korea, Goyang-si 410-769, South Korea.

${ }^{3}$ Department of Pathology, Center for Prostate Cancer, 4Biometrics Research Branch and Biostatistics Collaboration Unit, National Cancer Center, Goyang-si 410-769, South Korea.

The objective of this study was to evaluate the role of epithelial splicing regulatory protein 1 (ESRP1) expression in predicting prognosis and disease progression in a large cohort of PC patients with long- 
term follow-up. The ESRP1 participates in the epithelial-mesenchymal transition, a necessary prelude to disease progression in prostate cancer (PC). The prognostic role of ESRP1 has been studied in various human primary tumor tissues and has been described to be tumor-specific. However, little is known about the prognostic value of ESPR1 in PC. A preliminary investigation into the clinical significance of ESRP1 was conducted using The Cancer Genome Atlas PC dataset. Tissue microarrays of radical prostatectomy specimens from 514 PC patients at the National Cancer Center of Korea were immunohistochemically stained for ESRP1. PC samples were grouped into high and low expression of ESRP1 based on the immunohistochemistry results. The median follow-up period was 91.2 months. The immunohistochemistry was interpreted semi-quantitatively using $H$-score, defined by the intensity ( $0,1,2$ and 3 ), and the percentage of expressed areas (0-100\%). The prognostic significance of ESRP1 expression was analyzed using the Cox proportional-hazards model $(P<0.05)$. After adjusting for clinicopathological variables, high expression of ESRP1 was significantly associated with worse biochemical recurrence-free survival [hazard ratio (HR): 1.37 ; 95\% confidence interval $(\mathrm{CI}): 1.02-1.83 ; P=0.037$ ] and shorter cancer-specific survival (HR: 3.43; 95\% CI: 1.12-10.54; $P=0.031)$. PC patients with high expression of ESRP1 appear to have increased risk of biochemical recurrence and cancer-specific death. The expression of ESRP1 in patients with poor survival outcomes indicates that there may be an opportunity to predict response to androgen deprivation therapy based on ESRP1 expression.

\section{Biography}

Dr. Kang Hyun Lee has completed his MD and PhD from Seoul National University, College of Medicine, Seoul, Korea. He is a Tenure Chief Scientist in the Division of Precision Medicine and Specialist in Center for Prostate cancer, National Cancer Center of Korea. He is also the Emeritus Professor of GCSP and he has published more than 130 papers in academic journals and had been serving as 6th President of National Cancer Center of Korea.

\section{Gene-specific methylation as a molecular biomarker in gastric cancer}

Fernanda Wisnieski ${ }^{1}$, Leonardo Caires Santos ${ }^{1}$, Jaqueline Cruz Geraldis ${ }^{1}$, Mariana Ferreira Leal ${ }^{1}$, Ana Carolina Anauate Pereira ${ }^{1}$, Danielle Queiroz Calcagno ${ }^{2}$, Carolina Oliveira Gigek ${ }^{1}$, Elizabeth Suchi Chen ${ }^{1}$, Sâmia Demachki ${ }^{2}$, Ricardo Artigiani ${ }^{3}$, Paulo Pimentel Assumpção ${ }^{2}$, Laércio Gomes Lourenço ${ }^{4}$, Rommel Rodríguez Burbano ${ }^{5}$, Marília Cardoso Smith ${ }^{1}$

\footnotetext{
${ }^{1}$ Disciplina de Genética, Departamento de Morfologia e Genética, Universidade Federal de São Paulo, Sao Paolo 04021-001, Brazil.

${ }^{2}$ Núcleo de Pesquisas em Oncologia, Hospital Universitário João de Barros Barreto, Guamá, Belem 66073ooo, Brazil.

${ }^{3}$ Departamento de Patologia, Universidade Federal de São Paulo, Sao Paolo 04021-001, Brazil.

${ }^{4}$ Disciplina de Gastroenterologia Cirúrgica, Departamento de Cirurgia, Universidade Federal de São Paulo, Sao Paolo 04021-001, Brazil.

${ }^{5}$ Instituto de Ciências Biológicas, Universidade Federal do Pará, Guamá, Belem 66073-000, Brazil.
}

Despite the fact that overall rates of gastric cancer (GC) continue to decline worldwide, the majority of patients are still diagnosed with advanced disease in Western countries. New strategies for early diagnosis and new therapeutic methods in GC need to be explored. Epigenetic control using inhibitors of DNA methylation, such as 5-aza-2'-deoxycytidine (also known as Decitabine), may offer new possibilities in GC therapy. Our research group previously identified 86 differentially expressed genes (DEGs) by microarray analysis comparing GC cell lines treated with Decitabine and non-treated cells. Among the upregulated 
DEGs identified by this methodology, NRN1 was selected for further analyzes. This study aimed to evaluate, compare and correlate NRN1 mRNA and methylation levels of GC and adjacent non tumor samples from patients with primary gastric adenocarcinoma. The mRNA level was assessed by quantitative reverse transcription PCR. DNA methylation analysis was assessed by Ion Torrent PGM sequencer. A total of 12 CpG sites were evaluated in NRN1 promoter region. GC samples showed a significant increased DNA methylation at five specific $\mathrm{CpG}$ sites of $\mathrm{NRN}_{1}$ promotor region compared with adjacent non tumor samples $(P<0.05)$, besides no difference in NRN1 mRNA levels between these groups. At this time, no significant correlation between mRNA and methylation levels, as well as, significant clinicopathological associations were observed. Although different mechanisms may be involved in gene regulation, NRN1 methylation seems to play an important role in gastric carcinogenesis. This study is still in progress and new samples and other gene regions will be incorporated in our analyzes.

\section{Biography}

Fernanda Wisnieski has completed her PhD at Federal University of São Paulo (UNIFESP), São Paulo, Brazil. Currently she is a postdoctoral researcher at Genetics Division of UNIFESP. She has experience in the area of Human and Medical Genetics, working mainly on genetics and epigenetics aspects of gastric cancer clinical samples and cell lines models. Since the end of her doctorate degree she has published 15 papers in the area.

\section{A semi-comprehensive analysis of gene amplification in breast cancers using multiplex ligation-dependent probe amplification and fluorescence in situ hybridization}

\section{Akishi Ooi}

Kanazawa University, Kanazawa 920-1192, Japan.

Gene amplification is a common event in breast cancer. The aim of the present study was to determine the amplification status of 22 genes that are reportedly frequently amplified in breast cancers. A total of 320 formalin-fixed and paraffin-embedded invasive ductal cancer tissues were screened by multiple ligationdependent probe amplification, and 885 genes with "gain" or "amplified" status were further examined for the respective gene amplification by fluorescence in situ hybridization (FISH). In the FISH analysis, 116 of 320 tumors (36\%) displayed gene amplification of at least 1 of the 22 genes. The frequencies of gene amplification were as follows: ESR1 (5 cases, 1.5\%), EGFR (one case, 0.3\%), ZNF703 (25 cases, 7.8\%), FGFR1 (24 cases, 7.5\%), ADAM9 (17 cases, 5.3\%), IKBKB (10 cases, 3.1\%), PRDM1 (5 cases, $1.5 \%$ ), MTDH (15 cases, 4.7\% ), MYC (29 cases, 9.0\%), CCND1 (39 cases, 12\%), C11ORF30 (19 cases, 5.9\%), CDH1 (1 case, 0.3\%), CPD (9 cases, 2.8\%), MED1 (25 cases, 7.8\% ), ERBB2 ( 35 cases, $11 \%$ ), CDC6 (14 cases, $4.3 \%$ ), TOP2A (12 cases, 3.8\%), MAPT (1 case, 0.3\%), PPM1D (10 cases, 3\%), BIRC5 (6 cases, 1.9\%), CCNE1 (3 cases, 0.9\%), and AURKA ( 1 case, $0.3 \%$ ). In addition to the frequent co-amplifications of genes closely located on the same chromosome region, such as $8 \mathrm{p} 11,8 \mathrm{q} 24,11 \mathrm{q} 13$, or 17q12-21, co-amplification of genes located in different chromosomes was also found. The co-amplification of ZNF703/FGFR1 (8p11) and CCND1 (11q13) in single cells was found in 11 tumors, occurring in the same amplicons in 5. The co-amplification of ZNF703/FGFR1 and ERBB2 (17q12-21) in single tumor nuclei but different amplicons was observed in 11 cases, and that of CCND1 and ERBB2 was noted in 5 cases. The co-amplification of MYC (8q24) with $C C N D 1$ or $E R B B 2$ in single nuclei was found in three and two tumors, respectively. The amplifications of $E S R 1$ and $E R B B 2$ as well as EGFR and ERBB2 were found in one tumor each, although in different cancer cells. Amplified genes are attractive potential targets for therapy, so a precise and feasible analysis of the gene amplification status is clinically valuable. 


\section{Spinless metal isotopes are able to suppress DNA Polymerase Beta in WERI/Y79 retinoblastoma} cells: beyond the cytostatic effect

\section{Kirill V. Ermakov, Alexander A. Bukhvostov, Anton S. Dvornikov, Dmitry A. Kuznetsov}

Department of Medicinal NanoBioTechnologies, School of Medicine, N. I. Pirogov Russian National Research Medical University, Moscow 117997, Russian Federation.

Nuclear spin possessing isotopes $(25 \mathrm{Mg}, 43 \mathrm{Ca}, 67 \mathrm{Zn})$ promote the marked magnetic isotope effects (MIE) on DNA Polymerase Beta (DNApolB) in ex vivo survived human retinoblastoma (RB) cells. In $N$-ethylmelamide chase experiment, the RB in situ catalytic activity of DNApolB has been selectively estimat as a function of MIE. A resulted enzyme function breakdown leads to a sharp decrease of cancer cell viability. This study a paramagnetic chemotherapy path is all about. 